\title{
TOPOLOGICAL AND ALGEBRAIC REDUCIBILITY FOR PATTERNS ON TREES
}

\author{
LLUÍS ALSEDÀ, DAVID JUHER AND FRANCESC MAÑOSAS
}

\begin{abstract}
We extend the classical notion of block structure for periodic orbits of interval maps to the setting of tree maps and study the algebraic properties of the Markov matrix of a periodic tree pattern having a block structure. We also prove a formula which relates the topological entropy of a pattern having a block structure with that of the underlying periodic pattern obtained by collapsing each block to a point, and characterize the structure of the zero entropy patterns in terms of block structures. Finally, we prove that an $n$-periodic pattern has zero (positive) entropy if and only if all $n$-periodic patterns obtained by considering the $k$-th iterate of the map on the invariant set have zero (respectively, positive) entropy, for each $k$ relatively prime to $n$.
\end{abstract}

\section{Introduction, DEFINITIONS AND STATEMENT OF THE MAIN RESULTS}

The notion of reducibility plays a fundamental role in the study of the combinatorial and topological dynamics of discrete dynamical systems. Reducible systems are those such that the space can be decomposed in connected pieces with pairwise disjoint interiors which are permuted by the map. In this situation the behaviour of the original map can be related with the dynamics of an iterate of the map on the reduced pieces. This approach plays a crucial role, for instance in the study of both the surface homeomorphisms [13] and the interval dynamics related to periodic orbits (where reducibility is formalized through the notion of block structure) [4]. In this setting the concept of minimal dynamics plays also a fundamental role. Each minimal model has an associated (Markov) matrix that encodes at the algebraic level all its dynamical features, such as implications between periodic orbits, topological entropy, etc.

The aim of this paper is to clarify in full the notions of reducibility and irreducibility for periodic orbits of tree maps. We provide precise definitions of these notions for periodic patterns of trees and study the dynamical implications of these notions at a topological level. Moreover relate these features with the algebraic properties of the Markov matrices of the associated minimal models. Thanks to this study, we obtain some interesting properties of the topological entropy of reducible systems and we clarify its relation with the decomposition of the space and the Markov matrix of the map.

Before defining and study these notions we have to introduce the notation and definitions to fix the framework.

A tree is a compact uniquely arcwise connected space which is a point or a union of a finite number of intervals (by an interval we mean any space homeomorphic to $[0,1])$. Any continuous map $f: T \longrightarrow T$ from a tree $T$ into itself will be called a tree map. A set $X \subset T$ will be called $f$-invariant if $f(X) \subset X$. For each $x \in T$, we define the valence of $x$ to be the number of connected components of $T \backslash\{x\}$. A

1991 Mathematics Subject Classification. Primary: 37E25.

Key words and phrases. tree maps, patterns, topological entropy, block structure.

The authors have been partially supported by MEC grant numbers MTM2008-01486 and MTM2011-26995-C02-01. 
point of valence different from 2 will be called a vertex of $T$ and the set of vertices of $T$ will be denoted by $V(T)$. Each point of valence 1 will be called an endpoint of $T$. The set of such points will be denoted by $\operatorname{En}(T)$. The points in $V(T) \backslash \operatorname{En}(T)$ have valence greater than or equal to 3 . They will be called the branching points of $T$. Also, the closure of a connected component of $T \backslash V(T)$ will be called an edge of $T$.

Any tree which is a union of $r \geq 2$ intervals whose intersection is a unique point $y$ of valence $r$ will be called an $r$-star, and $y$ will be called its central point.

Given any subset $X$ of a topological space, we will denote by $\operatorname{Int}(X)$ and $\mathrm{Cl}(X)$ the interior and the closure of $X$, respectively. For a finite set $P$ we will denote its cardinality by $|P|$.

A triplet $(T, P, f)$ will be called a model if $f: T \longrightarrow T$ is a tree map and $P$ is a finite $f$-invariant set. In particular, if $P$ is a periodic orbit of $f$ and $|P|=n$ then $(T, P, f)$ will be called an $n$-periodic model. Given $X \subset T$ we will define the convex hull of $X$, denoted by $\langle X\rangle_{T}$ or simply by $\langle X\rangle$, as the smallest closed connected subset of $T$ containing $X$. When $X=\{x, y\}$ we will write $\langle x, y\rangle$ or $[x, y]$ to denote $\langle X\rangle$. The notations $(x, y),(x, y]$ and $[x, y)$ will be understood in the natural way.

Let $T$ be a tree and let $P \subset T$ be a finite subset of $T$. The pair $(T, P)$ will be called a pointed tree. A set $Q \subset P$ is said to be a discrete component of $(T, P)$ if either $|Q|>1$ and there is a connected component $C$ of $T \backslash P$ such that $Q=\mathrm{Cl}(C) \cap P$, or $|Q|=1$ and $Q=P$. We say that two pointed trees $(T, P)$ and $\left(T^{\prime}, P^{\prime}\right)$ are equivalent if there exists a bijection $\phi: P \longrightarrow P^{\prime}$ which preserves discrete components. In this case, two discrete components $C$ of $(T, P)$ and $C^{\prime}$ of $\left(T^{\prime}, P^{\prime}\right)$ will be called equivalent if $C^{\prime}=\phi(C)$. The equivalence class of a pointed tree $(T, P)$ will be denoted by $[T, P]$, and the equivalence class of a discrete component of $(T, P)$ will be called a discrete component of $[T, P]$.

Let $(T, P)$ and $\left(T^{\prime}, P^{\prime}\right)$ be equivalent pointed trees, and let $\theta: P \longrightarrow P$ and $\theta^{\prime}: P^{\prime} \longrightarrow P^{\prime}$ be maps. We will say that $\theta$ and $\theta^{\prime}$ are equivalent if $\theta^{\prime}=\varphi \circ \theta \circ \varphi^{-1}$ for a bijection $\varphi: P \longrightarrow P^{\prime}$ which preserves discrete components. The equivalence class of $\theta$ by this relation will be denoted by $[\theta]$. If $[T, P]$ is an equivalence class of pointed trees and $[\theta]$ is an equivalence class of maps then the pair $([T, P],[\theta])$ will be called a pattern. Any discrete component of $[T, P]$ will be also called a discrete component of the pattern $([T, P],[\theta])$.

We say that a model $(T, P, f)$ exhibits a pattern $(\mathcal{T}, \Theta)$ if $\mathcal{T}=\left[\langle P\rangle_{T}, P\right]$ and $\Theta=\left[\left.f\right|_{P}\right]$. Alternatively, we will say that the model $(T, P, f)$ is a representative of the pattern $(\mathcal{T}, \Theta)$.

The topological entropy [1] is a well known quantitative measure of the dynamical complexity of a model. It is an important topological invariant which is defined for continuous maps on compact metric spaces. The topological entropy of a map $f: T \longrightarrow T$ will be denoted by $h(f)$. Given a pattern $\mathcal{P}$, the topological entropy of $\mathcal{P}$ is defined to be

$$
h(\mathcal{P}):=\inf \{h(f):(T, P, f) \text { is a model exhibiting } \mathcal{P}\} .
$$

The simplest models exhibiting a given pattern are the monotone ones, according to the following definition. Let $S$ and $T$ be trees and let $f: T \longrightarrow S$ be a map. Given $a, b \in T$ we say that $\left.f\right|_{[a, b]}$ is monotone if $f([a, b])$ is either an interval or a point and $\left.f\right|_{[a, b]}$ is monotone as an interval map. Let $(T, P, f)$ be a model. A pair $\{a, b\} \subset P$ will be called a basic path of $(T, P)$ if it is contained in a single discrete component of $(T, P)$. We will say that $f$ is $P$-monotone if $\operatorname{En}(T) \subset P$ and $\left.f\right|_{[a, b]}$ is monotone for any basic path $\{a, b\}$. The model $(T, P, f)$ will be called monotone. In such case, Proposition 4.2 of [4] states that the set $P \cup V(T)$ is $f$-invariant. It easily follows that the map $f$, which is $P$-monotone, is also $(P \cup V(T))$-monotone. Observe that 
the closure of each connected component of $T \backslash(P \cup V(T))$ is an interval $[v, w]$, which is mapped by $f$ either to a point or to the proper interval $[f(v), f(w)]$. In the latter case, of course we can assume without loss of generality that $\left.f\right|_{[v, w]}$ is injective. For practical purposes, all monotone models considered throughout this paper will be assumed to satisfy this additional property.

Theorem 1.1 (Theorem A of [3]). Let $\mathcal{P}$ be a pattern. Then the following statements hold.

(a) There exists a monotone model of $\mathcal{P}$.

(b) Every monotone model $(T, P, f)$ of $\mathcal{P}$ satisfies $h(f)=h(\mathcal{P})$.

The monotone models from Theorem 1.1 are essentially unique in the following sense. Let $(T, P, f)$ be a monotone model and let $S$ be a non-empty union of edges disjoint from $P$. We will say that $S$ is an invariant forest of $(T, P, f)$ if either $f^{i}(S) \cap P=\emptyset$ for every $i \geq 0$ or there exists $n>0$ such that $f^{i}(S) \cap P=\emptyset$ for every $i=0,1, \ldots, n-1$ and $f^{n}(S)$ degenerates to a point of $P$. We will say that $(T, P, f)$ is a canonical model of the pattern $[T, P, f]$ if it has no invariant forests. From $[3$, Theorem B] it follows that every pattern has a canonical model. Moreover, given two canonical models $(T, P, f)$ and $\left(T^{\prime}, P^{\prime}, f^{\prime}\right)$ of the same pattern there exists a homeomorphism $\varphi: T \longrightarrow T^{\prime}$ such that $\varphi(P)=P^{\prime}$, and $\left.f^{\prime} \circ \varphi\right|_{P}=\left.\varphi \circ f\right|_{P}$. Hence, the canonical model of a pattern is essentially unique.

It is worth noticing that the proof of Theorem 1.1 is constructive and gives a finite algorithm to construct the canonical model of any pattern.

To state the first main result of this paper we need to introduce the notions of trivial pattern, collapsing interval, Markov matrix, block structure and rotational structure, which depend only on the combinatorial data of the pattern.

An $n$-periodic pattern $\mathcal{P}$ will be called trivial if it has only one discrete component. In this case, for $n \geq 2$, let $(T, P)$ be a pointed tree such that $T$ is an $n$-star with $\operatorname{En}(T)=P=\left\{x_{1}, x_{2}, \ldots, x_{n}\right\}$ and let $y$ be its central point. Consider a rigid rotation on $T$, that is, a model $(T, P, f)$ such that $f(y)=y$ and $f$ maps bijectively $\left[y, x_{i}\right]$ onto $\left[y, x_{i+1}\right]$ for $1 \leq i<n$ and $\left[y, x_{n}\right]$ onto $\left[y, x_{1}\right]$. Clearly, $(T, P, f)$ is a monotone model with no invariant forests. In consequence, $(T, P, f)$ is the canonical model of $\mathcal{P}$. Therefore, it easily follows that every trivial pattern has entropy 0 .

Let $\mathcal{P}$ be a periodic pattern and let $(T, P, f)$ be the canonical model of $\mathcal{P}$. Any $(P \cup V(T))$-basic interval $[a, b]$ such that $f([a, b])$ reduces to a point will be called a collapsing interval of $\mathcal{P}$. Note that, in this case, since $\mathcal{P}$ is periodic, $\{a, b\} \not \subset P$. On the other hand, since $(T, P, f)$ has no invariant forests, $\{a, b\} \not \subset V(T) \backslash P$. Therefore, each collapsing interval has the form $[a, b]$ with $a \in P$ and $b \in V(T) \backslash P$. The interval $[c, 8]$ in the canonical model of the pattern $\mathcal{P}$ shown in Figure 4 is an example of a collapsing interval, since $f(c)=f(8)=9$.

It should be mentioned that there is a purely combinatorial criterion to decide whether a pattern $\mathcal{P}$ has collapsing intervals without constructing its canonical model. Indeed, it is easy to see that a pattern $([T, P],[f])$ has collapsing intervals if and only if there is a discrete component $C$ of $(T, P)$ such that $|\operatorname{En}(\langle f(C)\rangle)|<|C|$. This definition is independent from the particular model $(T, P, f)$ realizing the pattern. For an example, recall that $[c, 8]$ is a collapsing interval for the pattern $\mathcal{P}$ in Figure 4 and observe that there is a discrete component $C=\{2,4,8,12\}$ such that $\langle f(C)\rangle=\langle\{3,5,9,1\}\rangle$, a tree with 3 endpoints. For another example of a pattern with collapsing intervals, see Figure 1: take the discrete component $C=\{1,5,13\}$ and observe that $\langle f(C)\rangle=\langle\{2,6,14\}\rangle$ is an interval.

Let $(T, Q, f)$ be a monotone model such that $Q \supset V(T)$. Note that, in this case, any connected component of $T \backslash Q$ is an open interval. An interval of $T$ will be 
called $Q$-basic if it is the closure of a connected component of $T \backslash Q$. Observe that two different $Q$-basic intervals have pairwise disjoint interiors. Given $K, L \subset T$, we will say that $K f$-covers $L$ if $f(K) \supset L$. Consider a labelling $I_{1}, I_{2}, \ldots I_{k}$ of all $Q$-basic intervals. The Markov graph of $(T, Q, f)$ associated to this labelling is a combinatorial directed graph whose vertices are the $Q$-basic intervals and there is an arrow from $I_{i}$ to $I_{j}$ if and only if $I_{i} f$-covers $I_{j}$. On the other hand, the Markov matrix of $(T, Q, f)$ associated to this labelling is a $k \times k$ matrix $\left(m_{i, j}\right)_{i, j=1}^{k}$ such that $m_{i, j}=1$ if and only if $I_{i} f$-covers $I_{j}$, and $m_{i, j}=0$ otherwise. Given two different labellings of the set of $Q$-basic intervals and their associated Markov matrices $M$ and $N$, there exists a permutation matrix $A$ such that $M=A^{T} N A$ (where $A^{T}$ denotes the transpose of $A$ ), and the corresponding Markov graphs are isomorphic.

Recall that if $(T, P, f)$ is the canonical model of a pattern $\mathcal{P}$ then the model $(T, P \cup V(T), f)$ is monotone. Thus, we can consider their Markov graph and matrix. Since both objects depend only on the canonical model of $\mathcal{P}$, which is uniquely determined by the combinatorial data of the pattern $\mathcal{P}$, they will be respectively called Markov graph of $\mathcal{P}$ and Markov matrix of $\mathcal{P}$.

We recall [8] that a square matrix with non-negative entries is called reducible if there exists a permutation matrix $A$ such that

$$
A^{T} M A=\left(\begin{array}{cc}
M_{11} & 0 \\
M_{21} & M_{22}
\end{array}\right)
$$

where $M_{11}$ and $M_{22}$ are square matrices of sizes $l \times l$ and $m \times m(l, m \geq 1)$ respectively and 0 stands for the $l \times m$ matrix whose entries are all 0 . If there does not exist such $A$ then the matrix $M$ is called irreducible.

An irreducible matrix $M$ is called primitive if all powers $M^{n}$ are irreducible for $n \geq 2$. Otherwise $M$ is called imprimitive. It is well known [8, Theorem 8] that an irreducible matrix $M$ is primitive if and only if there exists $n \geq 1$ such that all the entries of $M^{n}$ are positive.

A square matrix with non-negative entries $M$ will be called cyclic if there exist $p \geq 2$ and a permutation matrix $A$ such that

$$
A^{T} M A=\left(\begin{array}{ccccc}
0 & M_{1} & 0 & \ldots & 0 \\
0 & 0 & M_{2} & & 0 \\
\vdots & & \ddots & \ddots & \vdots \\
0 & 0 & \ldots & 0 & M_{p-1} \\
M_{p} & 0 & \ldots & 0 & 0
\end{array}\right)
$$

where the diagonal 0 blocks are square (possibly with pairwise different sizes). Of course, the matrix of a cyclic permutation is cyclic.

Remark 1.2. Let $M$ be an irreducible matrix. It is well known [8] that $M$ is imprimitive if and only if $M$ is cyclic.

The Markov matrix of a trivial pattern is the simplest example of a cyclic matrix. Indeed, the following remark states that the Markov matrix of a trivial pattern is, in fact, a permutation matrix.

Remark 1.3. Recall that if $(T, P, f)$ is the canonical model of a trivial $n$-periodic pattern $\mathcal{P}$ with $n \geq 3$ then $T$ is an $n$-star with $\operatorname{En}(T)=P$ and $f(y)=y$, where $y$ is the central point of $T$. It is straightforward to check that the Markov matrix $M$ of $\mathcal{P}$ is the permutation matrix corresponding to the cyclic permutation $(2,3, \ldots, n, 1)$. In consequence, $M$ is cyclic.

We are interested in describing some algebraic properties of the Markov matrix of a periodic pattern in terms of purely combinatorial features of the pattern. Let $\mathcal{P}=([T, P],[f])$ be an $n$-periodic pattern with $n \geq 3$. For $n>p \geq 2$, we will 


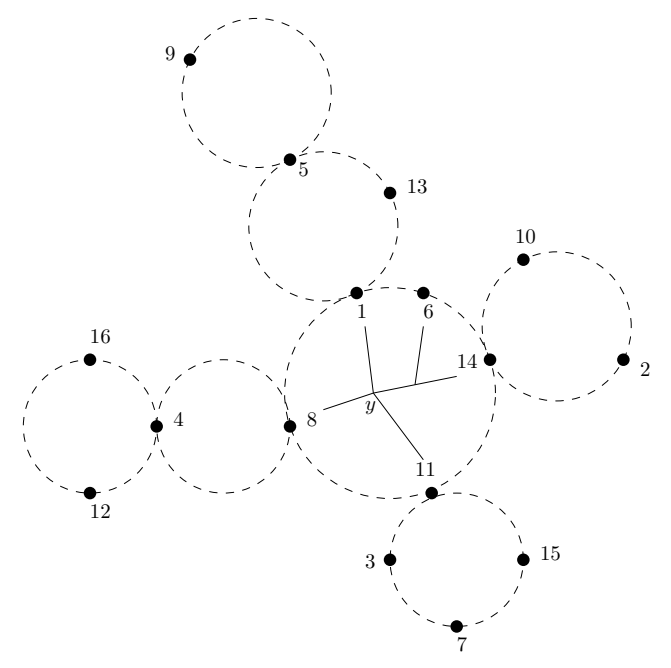

Figure 1. A 16-periodic pattern $\mathcal{P}=([T, P],[f])$. The dashed circles stand for the discrete components of $\mathcal{P}$. The points of $P$ are labelled with natural numbers, $f(i)=i+1$ for $1 \leq i<16$ and $f(16)=1$. The pointed tree corresponding to the discrete component $\{1,6,8,11,14\}$ in the canonical model is shown. The partition $P=P_{1} \cup P_{2}=\{1,3,5,7,9,11,13,15\} \cup\{2,4,6,8,10,12,14,16\}$ defines a 2-block structure for $\mathcal{P}$. There is also a 4-block structure given by the partition $P=Q_{1} \cup Q_{2} \cup Q_{3} \cup Q_{4}=\{1,5,9,13\} \cup$ $\{2,6,10,14\} \cup\{3,7,11,15\} \cup\{4,8,12,16\}$.

say that $\mathcal{P}$ has a p-block structure (or simply a block structure) if there exists a partition $P=P_{1} \cup P_{2} \cup \ldots \cup P_{p}$ such that $f\left(P_{i}\right)=P_{i+1}$ for $1 \leq i<p, f\left(P_{p}\right)=P_{1}$, and $\left\langle P_{i}\right\rangle_{T} \cap P_{j}=\emptyset$ whenever $i \neq j$. In this case, $p$ is a strict divisor of $n$ and $\left|P_{i}\right|=n / p$ for $1 \leq i \leq p$. Observe that from the equivalence relation which defines the class of models belonging to the pattern $\mathcal{P}$ it easily follows that this notion does not depend on the particular model $(T, P, f)$ representing $\mathcal{P}$. The trees $\left\langle P_{i}\right\rangle_{T}$ (which do depend on the particular model $(T, P, f)$ realizing the pattern) will be called blocks. See the pattern $\mathcal{P}$ in Figure 1 for an example: the partition $P=P_{1} \cup P_{2}=\{1,3,5,7,9,11,13,15\} \cup\{2,4,6,8,10,12,14,16\}$ defines a 2 -block structure for $\mathcal{P}$, since $\left\langle P_{1}\right\rangle_{T} \cap P_{2}=\left\langle P_{2}\right\rangle_{T} \cap P_{1}=\emptyset$ no matter what particular model $(T, P, f)$ represents $\mathcal{P}$.

We note that if a pattern has a $p$-block structure, this $p$-block structure is essentially unique up to relabelling of blocks. Observe also that a pattern can have several different block structures: see again Figure 1 for an example.

The existence of a block structure for a periodic pattern $\mathcal{P}$ is essentially equivalent to the fact that, for some $k \geq 1$, the $k$-th power $M^{k}$ of the Markov matrix $M$ of $\mathcal{P}$ is reducible (Corollary B). To look closer at the algebraic properties of $M$ (more precisely, to discriminate whether $M$ is reducible itself and to decide whether $M$ is cyclic) we need to define a couple of particular block structures, which we will respectively call separated structure and rotational structure. Let us introduce them.

Let $\mathcal{P}$ be an $n$-periodic pattern with $n \geq 3$ and let $(T, P, f)$ be the canonical model of $\mathcal{P}$. Assume that $\mathcal{P}$ has a $p$-block structure defined by a partition $P=$ $P_{1} \cup P_{2} \cup \ldots \cup P_{p}$. We say that this $p$-block structure is separated if $\left\langle P_{i}\right\rangle_{T} \cap\left\langle P_{j}\right\rangle_{T}=\emptyset$ whenever $i \neq j$. For instance, the 4-block structure $Q_{1} \cup Q_{2} \cup Q_{3} \cup Q_{4}$ for the pattern $\mathcal{P}$ in Figure 1 is separated, since the blocks have pairwise disjoint intersections in any 


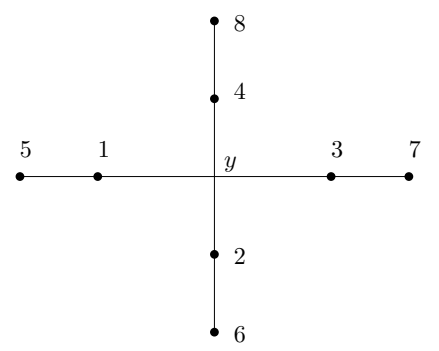

Figure 2. The canonical model $(T, P, f)$ of a 8 -periodic pattern $\mathcal{P}$, which satisfies $f(y)=y$. The pattern $\mathcal{P}$ has a separated and rotational 4-block structure given by the partition $\{1,5\} \cup\{2,6\} \cup$ $\{3,7\} \cup\{4,8\}$ and also a rotational 2-block structure given by the partition $\{1,3,5,7\} \cup\{2,4,6,8\}$, which is not separated.

model representing $\mathcal{P}$ (in particular, in the canonical model). On the other hand, a part of the tree $T$ corresponding to the canonical model is shown in Figure 1. Observe that $\left\langle P_{1}\right\rangle_{T} \cap\left\langle P_{2}\right\rangle_{T}=\{y\}$, where $y$ is a branching point of valence 4 . Therefore, the 2-block structure $P_{1} \cup P_{2}$ is not separated.

The cyclicity of the Markov matrix of a periodic pattern is related to the existence of another particular case of block structure. Let $\mathcal{P}$ be a non-trivial $n$-periodic pattern with $n \geq 3$ and let $(T, P, f)$ be the canonical model of $\mathcal{P}$. Assume that there exists a branching point $y \in T$ such that $f(y)=y$. For $n>p \geq 2$, we will say that $\mathcal{P}$ has a p-rotational structure (or simply a rotational structure) if there exist subtrees $Y_{1}, Y_{2}, \ldots, Y_{p}$ such that each $Y_{i}$ is the closure of a union of connected components of $T \backslash\{y\}, f\left(Y_{i}\right)=Y_{i+1}$ for $1 \leq i<p$ and $f\left(Y_{p}\right)=Y_{1}$. Note that the sets $Y_{i} \backslash\{y\}$ form a partition of $T \backslash\{y\}$. In this situation, we will see (Lemma 2.2) that the partition $P=P_{1} \cup P_{2} \cup \ldots \cup P_{p}$, where $P_{i}:=P \cap Y_{i}$ for $1 \leq i \leq p$, defines a $p$-block structure for $\mathcal{P}$. In other words, a rotational structure is a particular case of block structure. Moreover,

(a) Either all blocks $\left\langle P_{i}\right\rangle$ are pairwise disjoint,

(b) $\operatorname{Or}\left\langle P_{i}\right\rangle \cap\left\langle P_{j}\right\rangle=\{y\}$ whenever $i \neq j$.

Hence, a rotational structure is either separated or every pair of blocks in the canonical model intersect at a fixed branching point.

Of course, a pattern can have several different rotational structures. For an example, consider the 8-periodic pattern $\mathcal{P}$ whose canonical model $(T, P, f)$ is depicted in Figure 2. In this case, $y$ is the only branching point in $T$ and $f(y)=y$. The connected components of $T \backslash\{y\}$ are the intervals $(y, 5],(y, 6],(y, 7]$ and $(y, 8]$, whose closures which are mapped cyclically by $f$. Hence, $\{1,5\} \cup\{2,6\} \cup\{3,7\} \cup\{4,8\}$ defines a 4 -rotational structure for $\mathcal{P}$. Since the blocks $\langle 1,5\rangle,\langle 2,6\rangle,\langle 3,7\rangle,\langle 4,8\rangle$ are pairwise disjoint, (a) holds and this rotational structure is separated. On the other hand, since $f$ also maps cyclically the sets $[y, 5] \cup[y, 7]$ and $[y, 6] \cup[y, 8]$, the partition $\{1,3,5,7\} \cup\{2,4,6,8\}$ defines a 2 -rotational structure for $\mathcal{P}$, which is not separated because the blocks $\langle\{1,3,5,7\}\rangle$ and $\langle\{2,4,6,8\}\rangle$ intersect at $y$. Observe that the points of $P$ rotate around the discrete component $\{1,2,3,4\}$ under the action of $f$. This fact justifies the name rotational structure.

For another example, see the 12-periodic pattern whose canonical model $(T, P, f)$ is shown in Figure 4. In this case, there is only one fixed point $y$ of $f$, which belongs to $(1,10)$. Since $y$ is not a branching point, the two block structures exhibited by $\mathcal{P}$ are not rotational.

Note that there exist block structures that are not rotational neither separated. In Figure 3 we show a simple example: the 8 -periodic pattern $\mathcal{P}$ has no rotational 


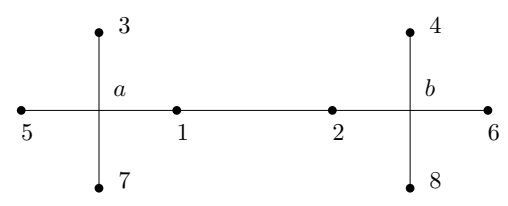

Figure 3. The canonical model $(T, P, f)$ of a 8 -periodic pattern $\mathcal{P}$, which satisfies $f(a)=b, f(b)=a$. The pattern $\mathcal{P}$ has a non-separated 4-block structure given by the partition $\{1,5\} \cup\{2,6\} \cup\{3,7\} \cup\{4,8\}$ and a separated 2-block structure given by $\{1,3,5,7\} \cup\{2,4,6,8\}$.

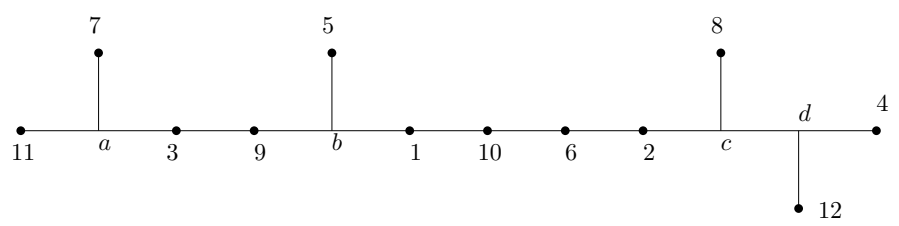

Figure 4. The canonical model $(T, P, f)$ of a 12-periodic pattern $\mathcal{P}$, which satisfies $f(a)=d, f(b)=6, f(c)=9, f(d)=b$. The pattern $\mathcal{P}$ has a separated 4-block structure given by the partition $\{1,5,9\} \cup\{2,6,10\} \cup\{3,7,11\} \cup\{4,8,12\}$ and a separated 2-block structure given by the partition $\{1,3,5,7,9,11\} \cup\{2,4,6,8,10,12\}$.

structures, since the only fixed point in the canonical model lies on the open interval $(1,2)$ and, in consequence, is not a branching point. On the other hand, the 4-block structure given by the partition $P_{1} \cup P_{2} \cup P_{3} \cup P_{4}=\{1,5\} \cup\{2,6\} \cup\{3,7\} \cup\{4,8\}$ is not separated. However, the pattern has also a separated 2-block structure given by the partition $\{1,3,5,7\} \cup\{2,4,6,8\}$, obtained by grouping together some sets $P_{i}$. This is, in fact, the idea of the next lemma which states that the the notions of separated and rotational structure are not restrictive when a pattern has a block structure. It will be proved in Section 2.

Lemma 1.4. Let $\mathcal{P}$ be a periodic pattern. Then, $\mathcal{P}$ has block structures if and only if $\mathcal{P}$ has rotational or separated block structures.

We remark that the notion of block structure is purely combinatorial, since it depends only on the discrete components of $\mathcal{P}$. In contrast, a block structure will be separated or rotational depending on some topological properties of the blocks in the canonical model of $\mathcal{P}$. However, since the canonical model is unique and it is constructed by means of a well defined algorithm uniquely determined by the combinatorial data of $\mathcal{P}$, in fact both notions are also intrinsic (in the sense that depend only on the combinatorial data of $\mathcal{P}$ ).

In the literature one can find several kinds of block structures and related notions for periodic orbits. In the interval case, the Sharkovskii's square root construction (see [12] or [4]) is an early example of a block structure. Also the notion of extension, first appeared in [7], gives rise to some particular cases of block structures for interval periodic orbits. Finally, the notion of division, introduced in [9] for interval periodic orbits and generalized in [2] and [5] in order to study the topological entropy and the set of periods for tree maps, has a strong connection with the notion of rotational structure.

Now we are ready to state the first main result of this paper.

Theorem A. Let $\mathcal{P}$ be an $n$-periodic pattern with $n \geq 3$ and let $M$ be the Markov matrix of $\mathcal{P}$. The following statements hold: 
(a) $M$ is reducible if and only if $\mathcal{P}$ has separated block structures or collapsing intervals.

(b) $M$ is cyclic if and only if either $\mathcal{P}$ is trivial or has rotational structures.

From Theorem A, Lemma 1.4 and Remark 1.2 we immediately get the following result.

Corollary B. Let $\mathcal{P}$ be a non-trivial n-periodic pattern with $n \geq 3$ and let $M$ be the Markov matrix of $\mathcal{P}$. Then, $M^{k}$ is reducible for some $k \geq 1$ if and only if $\mathcal{P}$ has collapsing intervals or block structures. Equivalently, $M$ is primitive if and only if $\mathcal{P}$ has no collapsing intervals and no block structures.

Let us see some examples. Consider again the 8-periodic pattern $\mathcal{P}$ shown in Figure 2. Recall that $\mathcal{P}$ has a 2-rotational structure. Therefore, by Theorem A(b), its Markov matrix is cyclic. Indeed, consider the following labelling of the set of $(P \cup V(T))$-basic intervals: $I_{1}=[1,5], I_{2}=[y, 1], I_{3}=[y, 3], I_{4}=[3,7], I_{5}=[2,6]$, $I_{6}=[y, 2], I_{7}=[y, 4], I_{8}=[4,8]$. Then, the Markov matrix $M$ of $\mathcal{P}$ associated to this labelling has the cyclic form

$$
\left(\begin{array}{cccc:cccc}
0 & 0 & 0 & 0 & 1 & 0 & 0 & 0 \\
0 & 0 & 0 & 0 & 0 & 1 & 0 & 0 \\
0 & 0 & 0 & 0 & 0 & 0 & 1 & 0 \\
0 & 0 & 0 & 0 & 0 & 0 & 0 & 1 \\
\hdashline 0 & 0 & 0 & 1 & 0 & 0 & 0 & 0 \\
0 & 0 & 1 & 0 & 0 & 0 & 0 & 0 \\
1 & 1 & 0 & 0 & 0 & 0 & 0 & 0 \\
1 & 0 & 0 & 0 & 0 & 0 & 0 & 0
\end{array}\right) .
$$

On the other hand, $\mathcal{P}$ has a separated 4-block structure. Therefore, by Theorem $\mathrm{A}(\mathrm{a}), M$ is reducible. Indeed, consider the labelling $J_{1}=[1,5], J_{2}=[2,6]$, $J_{3}=[3,7], J_{4}=[4,8], J_{5}=[y, 1], J_{6}=[y, 2], J_{7}=[y, 3], J_{8}=[y, 4]$. Then, the associated Markov matrix of $\mathcal{P}$ has the reducible form

$$
\left(\begin{array}{cccc:cccc}
0 & 1 & 0 & 0 & 0 & 0 & 0 & 0 \\
0 & 0 & 1 & 0 & 0 & 0 & 0 & 0 \\
0 & 0 & 0 & 1 & 0 & 0 & 0 & 0 \\
1 & 0 & 0 & 0 & 0 & 0 & 0 & 0 \\
\hdashline 0 & 0 & 0 & 0 & 0 & 1 & 0 & 0 \\
0 & 0 & 0 & 0 & 0 & 0 & 1 & 0 \\
0 & 0 & 0 & 0 & 0 & 0 & 0 & 1 \\
1 & 0 & 0 & 0 & 1 & 0 & 0 & 0
\end{array}\right) .
$$

It is worth noticing that Theorem A generalizes some well known results for interval patterns. It is folk knowledge that a periodic interval pattern has a block structure if and only if its Markov matrix is reducible (see, for instance, the proof of Lemma 4.4.16 of [4], where this fact is proved for a particular case of block structure called extension). In fact, this is true not just for interval patterns but for a broader class of patterns, which we call simplicial. A pattern $([T, P],[\theta])$ is called simplicial if each discrete component of $(T, P)$ has two points. Observe that, in this case, for each pointed tree $(S, Q) \in[T, P]$ we have that $V(S) \subset Q$ and, for each discrete component $C$ of $(S, Q),\langle C\rangle_{S}$ is an interval. For simplicial patterns (in particular, for interval patterns) the following simpler version of Theorem A holds:

Corollary C. Let $\mathcal{P}$ be a simplicial n-periodic pattern with $n \geq 3$ and let $M$ be the Markov matrix of $\mathcal{P}$. Then, $M$ is reducible if and only if $\mathcal{P}$ has a block structure. Moreover, if $M$ is irreducible then $M$ is primitive. 
The fourth main result of this paper has to do with the topological entropy of patterns with a block structure. It is a generalization of the following classical result for interval patterns. Take a periodic pattern on the interval (which can be identified with a cyclic permutation $\phi$ ) and a $P$-monotone interval map $f$ exhibiting $\phi$ over $P$. Assume that $\phi$ has a $p$-block structure defined by a partition $P=P_{1} \cup P_{2} \cup \ldots \cup P_{p}$. It is well known (Section 2.10 of [4]) that each block can be collapsed to a point in order to get a $p$-periodic pattern $\theta$ (see the details below) that we will call the skeleton of $\phi$. Assume in addition that $\left.\phi\right|_{P}$ is monotone on each block except at most one. This very particular type of block structure for interval patterns is called an extension [7]. In this situation, it is well known (Lemma 4.4 .16 of [4]) that the entropies of $\phi$ and $\theta$ are related via the following formula:

$$
h(\phi)=\max \left\{h(\theta),(1 / p) h\left(\left.f^{p}\right|_{\left\langle P_{i}\right\rangle}\right)\right\} \text { for any } 1 \leq i \leq p,
$$

which makes sense since $f^{p}$ maps every block $\left\langle P_{i}\right\rangle$ onto itself. Moreover, all the entropies $h\left(\left.f^{p}\right|_{\left\langle P_{i}\right\rangle}\right)$ are the same.

As far as we know, the above formula is just proven for extensions and there does not exist a similar result for interval patterns with a general $p$-block structure. The fourth main result of this paper generalizes the above formula to the setting of tree patterns with either a rotational structure or a separated block structure, with no additional restrictions (recall that, by Lemma 1.4, every pattern with block structures has either a rotational or a separated structure). In order to state it we need to introduce the notion of skeleton.

Let $\mathcal{P}$ be an $n$-periodic pattern and let $(T, P, f)$ be the canonical model of $\mathcal{P}$. Let $P=P_{1} \cup P_{2} \cup \ldots \cup P_{p}$ be a partition of $P$ which defines a separated $p$-block structure or a $p$-rotational structure for $\mathcal{P}$. We will see (Lemma 2.1) that in both cases $f\left(\left\langle P_{i}\right\rangle\right)=\left\langle P_{i+1}\right\rangle$ for $1 \leq i<p$ and $f\left(\left\langle P_{p}\right\rangle\right)=\left\langle P_{1}\right\rangle$. The skeleton of $\mathcal{P}$ (associated to this partition of $P$ ) is a $p$-periodic pattern $\mathcal{Q}$ defined as follows:

(a) If $P_{1} \cup P_{2} \cup \ldots \cup P_{p}$ defines a $p$-rotational structure for $\mathcal{P}$, then $\mathcal{Q}$ is defined to be a trivial $p$-periodic pattern.

(b) If $P_{1} \cup P_{2} \cup \ldots \cup P_{p}$ defines a separated $p$-block structure for $\mathcal{P}$ which is not a $p$-rotational structure, consider the tree $S$ obtained from $T$ by collapsing each block $\left\langle P_{i}\right\rangle$ to a point $x_{i}$. Let $\kappa: T \longrightarrow S$ be the standard projection, which is bijective on $T \backslash \cup_{i}\left\langle P_{i}\right\rangle$ and satisfies $\kappa\left(\left\langle P_{i}\right\rangle\right)=x_{i}$. Set $Q=\kappa(P)=\left\{x_{1}, x_{2}, \ldots, x_{p}\right\}$ and define $\theta: Q \longrightarrow Q$ by $\theta\left(x_{i}\right)=x_{i+1}$ for $1 \leq i<p$ and $\theta\left(x_{p}\right)=x_{1}$. Then the skeleton $\mathcal{Q}$ of $\mathcal{P}$ is defined to be the $p$-periodic pattern $([S, Q],[\theta])$. Observe that $\left.\theta \circ \kappa\right|_{P}=\left.\kappa \circ f\right|_{P}$.

Now we are ready to state the fourth main result of this paper.

Theorem D. Let $\mathcal{P}$ be an $n$-periodic pattern and let $(T, P, f)$ be the canonical model of $\mathcal{P}$. Assume that there is a partition $P=P_{1} \cup P_{2} \cup \ldots \cup P_{p}$ which defines either a p-rotational structure or a separated p-block structure for $\mathcal{P}$. Let $\mathcal{Q}$ be the associated skeleton of $\mathcal{P}$. Then, all the entropies $h\left(\left.f^{p}\right|_{\left\langle P_{i}\right\rangle}\right)$ are equal and

$$
h(\mathcal{P})=\max \left\{h(\mathcal{Q}),(1 / p) h\left(\left.f^{p}\right|_{\left\langle P_{i}\right\rangle}\right)\right\} \text { for any } 1 \leq i \leq p .
$$

The fifth main result of this paper describes the zero entropy periodic patterns (i.e. periodic patterns $\mathcal{P}$ such that $h(\mathcal{P})=0$ ) in terms of the existence of a very particular class of block structures. This result relies on Theorem $\mathrm{D}$ and on a nice characterization of the zero entropy patterns (not just those associated to periodic orbits) first given in [3]. We will recall the terminology and the results of [3] necessary to establish such a characterization in Section 5.

Let $\mathcal{P}=([T, P],[f])$ be a periodic pattern with a $p$-block structure defined by a partition $P=P_{1} \cup P_{2} \cup \ldots \cup P_{p}$. We will say that this $p$-block structure has trivial 

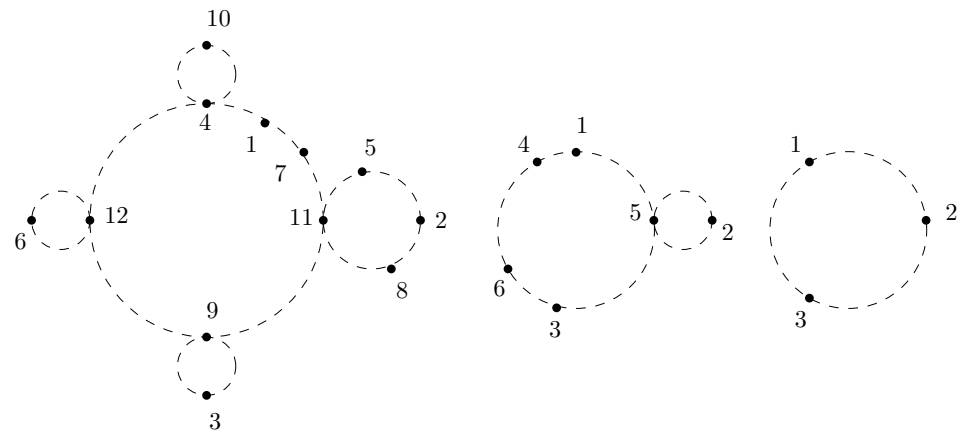

FiguRE 5. On the left, a 12-periodic pattern $\mathcal{P}_{3}$ with a separated 6-block structure $\{1,7\} \cup\{2,8\} \cup\{3,9\} \cup\{4,10\} \cup\{5,11\} \cup\{6,12\}$ with trivial blocks. The dashed circles stand for the discrete components of $\mathcal{P}$. In the centre, the corresponding skeleton $\mathcal{P}_{2}$, with a 3-block structure $\{1,4\} \cup\{2,5\} \cup\{3,6\}$ with trivial blocks. On the right, the corresponding skeleton $\mathcal{P}_{1}$, a trivial pattern.

blocks if the patterns $\left(\left[\left\langle P_{i}\right\rangle, P_{i}\right],\left[\left.f^{p}\right|_{P_{i}}\right]\right)$ are trivial for $1 \leq i \leq p$. Equivalently, $P_{i}$ is contained in a discrete component of $(T, P)$ for $1 \leq i \leq p$. Observe that this notion is independent from the particular model $(T, P, f)$ representing the pattern $\mathcal{P}$.

An $n$-periodic pattern $\mathcal{P}$ will be called 1 -starry if $\mathcal{P}$ is trivial. For $k \geq 2, \mathcal{P}$ will be called $k$-starry if $\mathcal{P}$ has a separated $p$-block structure with trivial blocks whose associated skeleton is $(k-1)$-starry. For an example, consider the 12 -periodic pattern $\mathcal{P}_{3}$ of Figure 5. By constructing the canonical model of $\mathcal{P}_{3}$ one checks that the block structure $\{1,7\} \cup\{2,8\} \cup\{3,9\} \cup\{4,10\} \cup\{5,11\} \cup\{6,12\}$, with trivial blocks, is separated. The associated skeleton $\mathcal{P}_{2}$ has also a separated 3-block structure $\{1,4\} \cup\{2,5\} \cup\{3,6\}$, again with trivial blocks. Finally, its associated skeleton $\mathcal{P}_{1}$ is a trivial pattern. Hence, the patterns $\mathcal{P}_{k}$ are $k$-starry for $k=1,2,3$.

Theorem E. A periodic pattern $\mathcal{P}$ has entropy zero if and only if $\mathcal{P}$ is $k$-starry for some $k \geq 1$.

Observe the recursive nature of Theorem E: the fact that an $n$-periodic pattern has entropy 0 is translated to the fact that a collection of periodic patterns (the skeleton and those associated to the blocks), with periods strictly smaller than $n$, have entropy 0 . It is well known that the same happens for interval periodic patterns (see, for instance, Corollary 4.4.19 of [4]). However, we emphasize that in order for $\mathcal{P}$ to have entropy 0 it is not enough that the patterns exhibited by $f^{p}$ on each block have entropy 0 . In addition, they must be trivial. This fact can be used to quickly discard that a given pattern has entropy 0. For an example, see the 12-periodic pattern $\mathcal{P}$ in Figure 6 . It has a unique block structure given by the partition $P_{1} \cup P_{2}=\{1,3,5,7,9,11\} \cup\{2,4,6,8,10,12\}$. Since the corresponding blocks are not trivial, $\mathcal{P}$ cannot be starry. In consequence, the entropy of $\mathcal{P}$ is positive by Theorem E.

Lastly, we prove a result that derives easily from Theorems D and E. We recall that if $([T, P],[f])$ is an $n$-periodic pattern and we take any $k \in \mathbb{N}$ such that $k$ and $n$ are relatively prime, then $\left([T, P],\left[f^{k}\right]\right)$ is also an $n$-periodic pattern (different from $([T, P],[f])$ in general).

Theorem $\mathbf{F}$. Let $\mathcal{P}=([T, P],[f])$ be an $n$-periodic pattern. Then:

(a) $\mathcal{P}$ has zero entropy if and only if all patterns $\left([T, P],\left[f^{k}\right]\right)$, for each $k \in \mathbb{N}$ such that $k$ and $n$ are relatively prime, have zero entropy. 


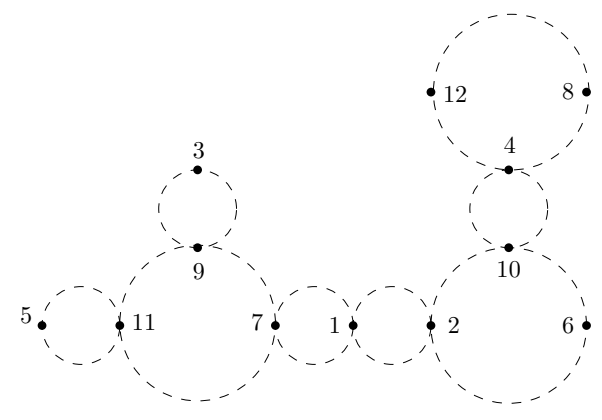

Figure 6. A 12-periodic pattern $\mathcal{P}$, which has a unique 2-block structure $P_{1} \cup P_{2}=\{1,3,5,7,9,11\} \cup\{2,4,6,8,10,12\}$. The associated skeleton is a trivial 2-periodic pattern, and the patterns $\left(\left[\left\langle P_{i}\right\rangle, P_{i}\right],\left[f^{2}\right]\right)$ have entropy 0 . However, $h(\mathcal{P}) \approx \log (1.98)>0$.

(b) $\mathcal{P}$ has positive entropy if and only if all patterns $\left([T, P],\left[f^{k}\right]\right)$, for each $k \in \mathbb{N}$ such that $k$ and $n$ are relatively prime, have positive entropy.

As far as we know, Theorem $\mathrm{F}$ was not explicitly stated in the literature, even for interval patterns. We also remark that in general the entropies of the patterns $\left([T, P],\left[f^{k}\right]\right)$ in the statement (b) of Theorem $\mathrm{F}$ need not be equal.

This paper is organized as follows. In Section 2 we prove some technical results on the topological distribution of the blocks in the canonical model of patterns with a block structure and prove Lemma 1.4. In Section 3 we prove Theorem A and Corollary C. Section 4 is devoted to prove Theorem D. Finally in Section 5 we introduce the notions and results from [3] which characterize the zero entropy patterns and use them to prove Theorems E and F.

\section{Rotational structures. Proof of Lemma 1.4}

The aim of this section is to study the properties of block structures and finally prove Lemma 1.4. We start with a simple lemma on the cyclicity of the blocks in the canonical model of a pattern with a block structure.

Lemma 2.1. Let $\mathcal{P}$ be a periodic pattern and let $(T, P, f)$ be the canonical model of $\mathcal{P}$. Assume that $\mathcal{P}$ has a $p$-block structure given by a partition $P=P_{1} \cup P_{2} \cup \ldots \cup P_{p}$. Then, $f\left(\left\langle P_{i}\right\rangle\right)=\left\langle P_{i+1}\right\rangle$ for $1 \leq i<p$ and $f\left(\left\langle P_{p}\right\rangle\right)=\left\langle P_{1}\right\rangle$.

Proof. By the definition of a $p$-block structure, $\left\langle P_{i}\right\rangle \cap P_{j}=\emptyset$ whenever $i \neq j$. Equivalently,

$$
\left\langle P_{k}\right\rangle \cap P=P_{k} \text { for } 1 \leq k \leq p .
$$

From (3) and the fact that $\left\langle P_{k}\right\rangle$ is the smallest connected set containing $P_{k}$, it follows that each $\left\langle P_{k}\right\rangle$ can be written as the union of all the intervals of the form $[a, b]$ such that $\{a, b\} \subset P_{k}$ is a basic path of $(T, P)$. Then, it is enough to see that $f([a, b]) \subset\left\langle P_{k+1}\right\rangle$ for such basic intervals $[a, b]$. Since $f$ is $P$-monotone, $f([a, b])=$ $[f(a), f(b)]$, which is obviously contained in $\left\langle P_{k+1}\right\rangle$ because $\{f(a), f(b)\} \subset P_{k+1}$.

The next result states that a rotational structure is a particular case of block structure. It also describes the spatial distribution of the corresponding blocks.

Lemma 2.2. Let $\mathcal{P}$ be an $n$-periodic pattern and let $(T, P, f)$ be the canonical model of $\mathcal{P}$. Assume that there exists a partition $P=P_{1} \cup P_{2} \cup \ldots \cup P_{p}$ which defines a $p$ rotational structure for $\mathcal{P}$. Then, the same partition defines a p-block structure for 
$\mathcal{P}$. Moreover, either this p-block structure is separated or there exists a branching point $y$ with $f(y)=y$ such that $\left\langle P_{i}\right\rangle \cap\left\langle P_{j}\right\rangle=\{y\}$ whenever $i \neq j$.

Proof. Until the end of this proof, all the subindices will be considered modulo $p$, and we will take $\{1,2, \ldots, p\}$ as the representatives of $\mathbb{Z} / p$. By the definition of a $p$-rotational structure, there exist a branching point $y \in T$ with $f(y)=y$ and subtrees $Y_{1}, Y_{2}, \ldots, Y_{p}$ with $n>p \geq 2$ such that each $Y_{i}$ is the closure of a union of connected components of $T \backslash\{y\}, f\left(Y_{i}\right)=Y_{i+1}$ for $1 \leq i<p$ and $f\left(Y_{p}\right)=Y_{1}$. Moreover, $P_{i}=Y_{i} \cap P$ for $1 \leq i \leq p$. We have that $f\left(P_{i}\right)=P_{i+1}$ for $1 \leq i \leq p$.

Since all sets $Y_{k}$ are closures of unions of connected components of $T \backslash\{y\}$, it immediately follows that

$$
\langle K\rangle \subset Y_{k} \text { for each } 1 \leq k \leq p \text { and every } K \subset Y_{k} .
$$

Now let us see that $P_{1} \cup P_{2} \cup \ldots \cup P_{p}$ defines a $p$-block structure for $\mathcal{P}$. Indeed: since $y \notin P \supset P_{l}$, then $\left\langle P_{k}\right\rangle \cap P_{l}=\left(\left\langle P_{k}\right\rangle \backslash\{y\}\right) \cap P_{l}$. By (4), $\left\langle P_{k}\right\rangle \subset Y_{k}$. Then, since $P_{l} \subset Y_{l}$ and $Y_{k} \cap Y_{l}=\{y\}$, it follows that $\left\langle P_{k}\right\rangle \cap P_{j}=\emptyset$ whenever $k \neq l$. In consequence, $P_{1} \cup P_{2} \cup \ldots \cup P_{p}$ defines a $p$-block structure for $\mathcal{P}$.

From Lemma 2.1 we get that

$$
f\left(\left\langle P_{k}\right\rangle\right)=\left\langle P_{k+1}\right\rangle \text { for } 1 \leq k \leq p .
$$

Assume first that no block $\left\langle P_{k}\right\rangle$ contains $y$. Since $Y_{i} \cap Y_{j}=\{y\}$ when $i \neq j$, from (4) it follows that $\left\langle P_{i}\right\rangle \cap\left\langle P_{j}\right\rangle=\emptyset$ for $i \neq j$. Hence, in this case the $p$-block structure defined by $P_{1} \cup P_{2} \cup \ldots \cup P_{p}$ is separated. On the other hand, assume that there is a block $\left\langle P_{k}\right\rangle$ containing $y$. Then, from (5) and the fact that $f(y)=y$ it follows that all blocks contain $y$. Thus, (4) and the fact that $Y_{i} \cap Y_{j}=\{y\}$ when $i \neq j$ yield that $\left\langle P_{i}\right\rangle \cap\left\langle P_{j}\right\rangle=\{y\}$ for $i \neq j$.

The following is a technical result on the pairwise intersections of blocks in the canonical model of a pattern with a general block structure.

Lemma 2.3. Let $(T, P, f)$ be the canonical model of a periodic pattern $\mathcal{P}$. Assume that $\mathcal{P}$ has a p-block structure given by $P=P_{1} \cup P_{2} \cup \ldots \cup P_{p}$. Then, $\left\langle P_{i}\right\rangle \cap\left\langle P_{j}\right\rangle$ is either empty or consists of a single point of $V(T) \backslash P$, whenever $i \neq j$.

Proof. Until the end of this proof, all the subindices will be considered modulo $p$, and we will take $\{1,2, \ldots, p\}$ as the representatives of $\mathbb{Z} / p$.

For any pair $\{k, l\} \subset\{1,2, \ldots, p\}$, set $X_{k, l}:=\left\langle P_{k}\right\rangle \cap\left\langle P_{l}\right\rangle$. From the definition of a $p$-block structure it easily follows that

$$
X_{k, l} \cap P=\emptyset \text { for any pair } k, l .
$$

We have to prove that $X_{i, j}$ is either empty or reduces to a single point of $V(T) \backslash P$. Assume, by way of contradiction, that $X_{i, j}$ contains more than one point. Then, $X_{i, j}$ is a non-empty union of edges of $T$ and, by (6), is disjoint from $P$. Since $f$ is $(P \cup V(T))$-monotone, $f\left(X_{i, j}\right)$ is either a point or a non-empty union of edges of $T$ disjoint from $P$ and, by Lemma 2.1 , it is contained in $X_{i+1, j+1}$. Iterating this argument yields that $f^{k}\left(X_{i, j}\right)$ is disjoint from $P$ for every $k \geq 0$. Therefore, $X_{i, j}$ is an invariant forest, in contradiction with the fact that $(T, P, f)$ is a canonical model. This proves that $X_{i, j}$ reduces to one point $z$.

Thus, to end the proof of the lemma we have to see that $z \in V(T)$. Otherwise, $\operatorname{Val}(z)=2$ and, by $(6), z \notin P$. Hence, there is a unique $(P \cup V(T))$-basic interval $I$ such that $z \in \operatorname{Int}(I)$. In this case, since $\left\langle P_{i}\right\rangle$ and $\left\langle P_{j}\right\rangle$ are convex hulls of subsets of $P$, we would have that $I \subset X_{i, j}$, in contradiction with the fact that $X_{i, j}=\{z\}$.

The iterative use of Lemma 2.3 leads to the following lemma, which is the key result in the proof of Lemma 1.4 . 
Lemma 2.4. Let $(T, P, f)$ be the canonical model of a periodic pattern $\mathcal{P}$ with a p-block structure given by $P=P_{1} \cup P_{2} \cup \ldots \cup P_{p}$. Assume that there exist at least two different blocks with non-empty intersection. Then, there exists a divisor $m \geq 1$ of $p$ and an $m$-periodic orbit $\left\{y, f(y), \ldots, f^{m-1}(y)\right\} \subset V(T) \backslash P$ such that

$$
f^{i}(y) \in \bigcap_{j=0}^{\frac{p}{m}-1}\left\langle P_{i+1+j m}\right\rangle \text { for } 0 \leq i<m .
$$

Proof. By Lemma 2.3, if two blocks $\left\langle P_{i}\right\rangle$ and $\left\langle P_{j}\right\rangle$ are not disjoint then its intersection reduces to a point $y \in V(T) \backslash P$. By Lemma 2.1, $f(y) \in\left\langle P_{i+1}\right\rangle \cap\left\langle P_{j+1}\right\rangle$. Again by Lemma 2.3, $f(y) \in V(T) \backslash P$. The lemma easily follows from a simple iterative argument.

Finally we prove Lemma 1.4 .

Proof of Lemma 1.4. The "if" part of the statement is trivial. Let us prove the "only if" part. Let $(T, P, f)$ be the canonical model of $\mathcal{P}$ and assume that $\mathcal{P}$ has a $p$-block structure given by a partition $P=P_{1} \cup P_{2} \cup \ldots \cup P_{p}$. We claim that if this $p$-block structure is not separated then either it is rotational or there exists an $m$-block structure for $\mathcal{P}$ for some $1<m<p$. It is clear that the lemma follows from the iterative use of this claim.

Let us prove the claim. Since the $p$-block structure is not separated, at least two different blocks are not disjoint. Then, by Lemma 2.4, there exists a divisor $m \geq 1$ of $p$ and an $m$-periodic orbit $\left\{y, f(y), \ldots, f^{m-1}(y)\right\} \subset V(T) \backslash P$ such that

$$
f^{i}(y) \in \bigcap_{j=0}^{\frac{p}{m}-1}\left\langle P_{i+1+j m}\right\rangle \text { for } 0 \leq i<m .
$$

If $m=1$, then $\bigcap_{i=1}^{p}\left\langle P_{i}\right\rangle=\{y\}$ for a fixed point $y \in V(T) \backslash P$. Since $\operatorname{En}(T) \subset P$, $T=\bigcup_{i=1}^{p}\left\langle P_{i}\right\rangle$. It easily follows that $T \backslash\{y\}$ is the disjoint union of the sets $\left\langle P_{i}\right\rangle \backslash\{y\}$, each being a union of connected components of $T \backslash\{y\}$. Thus, the $p$-block structure defined by $P_{1} \cup P_{2} \cup \ldots \cup P_{p}$ is rotational and the claim holds in this case.

Assume that $m>1$. Set

$$
\bar{P}_{i}:=\bigcup_{j=0}^{\frac{p}{m}-1} P_{i+j m} \text { for } 1 \leq i \leq m .
$$

Then, $f\left(\bar{P}_{i}\right)=\bar{P}_{i+1}$ for $1 \leq i<m$ and $f\left(\bar{P}_{m}\right)=\bar{P}_{1}$. To see that $\bar{P}_{1} \cup \bar{P}_{2} \cup \ldots \cup \bar{P}_{m}$ defines an $m$-block structure for $\mathcal{P}$ we have to show that $\left\langle\bar{P}_{r}\right\rangle \cap \bar{P}_{s}=\emptyset$ whenever $r \neq s$. Indeed: since all blocks $\left\langle P_{r+j m}\right\rangle$ intersect at a single point in $V(T) \backslash P$, it follows that

$$
\left\langle\bar{P}_{r}\right\rangle=\bigcup_{j=0}^{\frac{p}{m}-1}\left\langle P_{r+j m}\right\rangle .
$$

Moreover, each point of $P$ in $\left\langle\bar{P}_{r}\right\rangle$ belongs to a unique block $\left\langle P_{r+k m}\right\rangle$. Since $P_{1} \cup$ $P_{2} \cup \ldots \cup P_{p}$ defines a $p$-block structure for $\mathcal{P},\left\langle P_{r+k m}\right\rangle$ is disjoint from any set of the form $P_{s+j m}$ and, hence, from $\bar{P}_{s}$. Therefore, $P=\bar{P}_{1} \cup \bar{P}_{2} \cup \ldots \cup \bar{P}_{m}$ defines an $m$-block structure for $\mathcal{P}$ and the claim is proved.

\section{Proofs of Theorem A and Corollary C}

The next two results describe the algebraic structure of the Markov matrix of the periodic patterns exhibiting separated and rotational block structures respectively. We will use them in the proofs of Theorems A and C. Lemma 3.1 tells us that the Markov matrix of a periodic pattern with a separated block structure is reducible. 
Lemma 3.1. Let $\mathcal{P}$ be an $n$-periodic pattern and let $(T, P, f)$ be the canonical model of $\mathcal{P}$. Assume that there is a partition $P=P_{1} \cup P_{2} \cup \ldots \cup P_{p}$ which defines a separated $p$-block structure for $\mathcal{P}$. Let $z$ be the number of $(P \cup V(T))$-basic intervals, let $n_{i}$ be the number of $(P \cup V(T))$-basic intervals contained in $\left\langle P_{i}\right\rangle$ for $i=1,2, \ldots, p$ and let $q=n_{1}+n_{2}+\ldots+n_{p}$. Then, $q<z$ and there exists a labelling of the set of $(P \cup V(T))$-basic intervals such that the Markov matrix of $\mathcal{P}$ reads as

$$
\left(\begin{array}{cc}
M_{11} & 0 \\
M_{21} & M_{22}
\end{array}\right)
$$

where $M_{11}$ and $M_{22}$ are square matrices of sizes $q \times q$ and $(z-q) \times(z-q)$ respectively, and

$$
M_{11}=\left(\begin{array}{ccccc}
0 & M_{1} & 0 & \ldots & 0 \\
0 & 0 & M_{2} & & 0 \\
\vdots & & \ddots & \ddots & \vdots \\
0 & 0 & \ldots & 0 & M_{p-1} \\
M_{p} & 0 & \ldots & 0 & 0
\end{array}\right)
$$

where the diagonal 0 blocks are square, $M_{i}$ is an $n_{i} \times n_{i+1}$ matrix for $1 \leq i<p$ and $M_{p}$ is an $n_{p} \times n_{1}$ matrix.

Proof. Since all blocks $\left\langle P_{i}\right\rangle$ are pairwise disjoint, it follows that for each $(P \cup V(T))$ basic interval $I$ there exists at most one $1 \leq i \leq p$ such that $I \subset\left\langle P_{i}\right\rangle$. Thus, $q=n_{1}+n_{2}+\ldots+n_{p} \leq z$. Let us see that $q<z$. Set $X:=\cup_{i=1}^{p}\left\langle P_{i}\right\rangle$. Observe that $X$ is a union of $(P \cup V(T))$-basic intervals. Moreover, since $p \geq 2$ and all blocks are pairwise disjoint, $X$ is not connected. If follows that the closure of $T \backslash X$ contains at least one $(P \cup V(T))$-basic interval. In consequence, $q<z$.

Set $\widetilde{n}_{0}=0$ and $\widetilde{n}_{i}=n_{1}+n_{2}+\ldots+n_{i}$ for $1 \leq i<p$. By Lemma 2.1, $f\left(\left\langle P_{i}\right\rangle\right)=\left\langle P_{i+1}\right\rangle$ for $1 \leq i<p$ and $f\left(\left\langle P_{p}\right\rangle\right)=\left\langle P_{1}\right\rangle$. Then, it is straightforward to check that the lemma holds for a labelling $I_{1}, I_{2}, \ldots, I_{z}$ of the set of $(P \cup V(T))$-basic intervals such that $I_{j+\widetilde{n}_{i-1}} \subset\left\langle P_{i}\right\rangle$ for $i=1,2, \ldots, p$ and $1 \leq j \leq n_{i}$.

The next result is the analogous of Lemma 3.1 for rotational structures. It tells us that the Markov matrix of a pattern with a rotational structure is cyclic.

Lemma 3.2. Let $\mathcal{P}$ be an $n$-periodic pattern and let $(T, P, f)$ be the canonical model of $\mathcal{P}$. Assume that there is a partition $P=P_{1} \cup P_{2} \cup \ldots \cup P_{p}$ which defines a $p$ rotational structure for $\mathcal{P}$. Let $z$ be the number of $(P \cup V(T))$-basic intervals, let $n_{i}$ be the number of $(P \cup V(T))$-basic intervals contained in $\left\langle P_{i}\right\rangle$ for $i=1,2, \ldots, p$ and let $q=n_{1}+n_{2}+\ldots+n_{p}$. Then, there is a labelling of the set of $(P \cup V(T))$-basic intervals such that the Markov matrix of $\mathcal{P}$ reads as

$$
\left(\begin{array}{ccccc}
0 & M_{1} & 0 & \cdots & 0 \\
0 & 0 & M_{2} & & 0 \\
\vdots & & \ddots & \ddots & \vdots \\
0 & 0 & \cdots & 0 & M_{p-1} \\
M_{p} & 0 & \cdots & 0 & 0
\end{array}\right)
$$

where the diagonal 0 blocks are square. Moreover,

(a) If all blocks are pairwise disjoint, then $z=q+p, M_{i}$ is an $\left(n_{i}+1\right) \times\left(n_{i+1}+1\right)$ matrix for $1 \leq i<p$ and $M_{p}$ is an $\left(n_{p}+1\right) \times\left(n_{1}+1\right)$ matrix.

(b) If all blocks intersect at a single point, then $z=q, M_{i}$ is an $n_{i} \times n_{i+1}$ matrix for $1 \leq i<p$ and $M_{p}$ is an $n_{p} \times n_{1}$ matrix.

Proof. By definition of a $p$-rotational structure, there exists a branching point $y \in T$ with $f(y)=y$ and subtrees $Y_{1}, Y_{2}, \ldots, Y_{p}$ with $n>p \geq 2$ such that each $Y_{i}$ is the closure of a union of connected components of $T \backslash\{y\}, f\left(Y_{i}\right)=Y_{i+1}$ for $1 \leq i<p$ 
and $f\left(Y_{p}\right)=Y_{1}$. Moreover, $P_{i}=Y_{i} \cap P$ for $1 \leq i \leq p$. By Lemma 2.2, either all blocks are pairwise disjoint or their pairwise intersections reduce to $\{y\}$. On the other hand, by Lemma 2.2 a $p$-rotational structure is in particular a $p$-block structure. So, by Lemma 2.1, $f\left(\left\langle P_{i}\right\rangle\right)=\left\langle P_{i+1}\right\rangle$ for $1 \leq i<p$ and $f\left(\left\langle P_{p}\right\rangle\right)=\left\langle P_{1}\right\rangle$. Set $\widetilde{n}_{0}=0$ and $\widetilde{n}_{i}=n_{1}+n_{2}+\ldots+n_{i}$ for $1 \leq i<p$.

Assume first that all blocks intersect at $\{y\}$. Then, each $(P \cup V(T))$-basic interval is contained in one (and only one) block. It follows that $q=n_{1}+n_{2}+\ldots+n_{p}=z$. Moreover, it is straightforward to check that (b) holds for a labelling $I_{1}, I_{2}, \ldots, I_{z}$ of the set of $(P \cup V(T))$-basic intervals such that $I_{j+\widetilde{n}_{i-1}} \subset\left\langle P_{i}\right\rangle$ for $i=1,2, \ldots, p$ and $1 \leq j \leq n_{i}$.

Now assume that all blocks are pairwise disjoint. As in the proof of Lemma 2.2, we get that each block $\left\langle P_{j}\right\rangle$ intersects only one connected component of $T \backslash\{y\}$. Therefore, $T \backslash\{y\}$ has exactly $p$ connected components, whose closures are the subtrees $Y_{1}, Y_{2}, \ldots, Y_{p}$, and $\left\langle P_{i}\right\rangle \subset Y_{i}$ for $1 \leq i \leq p$. Since $\operatorname{En}(T) \cap Y_{i} \subset P \cap Y_{i}=P_{i}$, it easily follows that for each $1 \leq i \leq p$ there exists a unique point $a_{i} \in\left\langle P_{i}\right\rangle$ such that $\left(y, a_{i}\right) \cap(P \cup V(T))=\emptyset$. In other words, $\left[y, a_{i}\right]$ is a $(P \cup V(T))$-basic interval and the number of $(P \cup V(T))$-basic intervals contained in $Y_{i}$ is $n_{i}+1$. Moreover, since $f(y)=y$ and $f\left(a_{i}\right) \in\left\langle P_{i+1}\right\rangle$, the $P$-monotonicity of $f$ implies that $\left[y, a_{i}\right] f$-covers $\left[y, a_{i+1}\right]$. It is straightforward to check that (a) holds for a labelling $I_{1}, I_{2}, \ldots, I_{z}$ of the set of $(P \cup V(T))$-basic intervals such that $I_{i-1+j+\widetilde{n}_{i-1}} \subset\left\langle P_{i}\right\rangle \cup\left[y, a_{i}\right]$ for $i=1,2, \ldots, p$ and $1 \leq j \leq n_{i}+1$.

Since a rotational structure can be simultaneously a separated block structure (Lemma 2.2), in view of Lemmas 3.1 and 3.2, the Markov matrix of a pattern with a rotational structure can be reducible and cyclic at the same time.

Now we are ready to prove Theorem A.

Proof of Theorem A. Let $(T, P, f)$ be a canonical model of $\mathcal{P}$. Set $P_{V}=P \cup V(T)$ and let $z$ be the number of $P_{V}$-basic intervals, so that $M$ is a $z \times z$ matrix.

Let us prove (a). We start by proving the "if" part of the statement. Assume that $\mathcal{P}$ has collapsing intervals. Then, $M$ has $l \geq 1$ rows whose elements are all 0 . Hence, $M$ is reducible because, for an appropriate permutation matrix $A$, (1) holds for an $l \times l$ matrix $M_{11}$ whose entries are all 0 .

Now assume that $P=P_{1} \cup P_{2} \cup \ldots \cup P_{p}$ is a partition of $P$ that defines a separated $p$-block structure for $\mathcal{P}$. By Lemma 3.1, there is a labelling of the set of $P_{V}$-basic intervals such that $M$ reads as the right hand side of (1), where $M_{11}$ is a $q \times q$ matrix and $M_{21}$ has $q-z>0$ rows. Hence, $M$ is reducible.

Next we prove the "only if" part of (a). Since $M$ is reducible, there is a labelling $I_{1}, I_{2}, \ldots, I_{z}$ of the set of $P_{V}$-basic intervals such that the Markov matrix of $\mathcal{P}$ reads as the right hand side of (1), where $M_{11}$ and $M_{22}$ are square matrices of sizes $l \times l$ and $m \times m(l, m \geq 1)$ respectively and 0 stands for the $l \times m$ matrix whose entries are all 0 . In particular, the set $X:=\bigcup_{i=1}^{l} I_{i}$ is $f$-invariant. Now we assume that $\mathcal{P}$ has no collapsing intervals, and we will show that $\mathcal{P}$ has a separated block structure.

Since $l<z$, it follows that $T \backslash X \neq \emptyset$. Let us see that $X$ has at least two connected components. First we note that $P \cap X \neq \emptyset$ because, otherwise, $X$ would be an invariant forest for $f$ disjoint from $P$, in contradiction with the fact that $(T, P, f)$ is a canonical model. Now observe that, since $P \cap X \neq \emptyset$ and $X$ is $f$ invariant, $P \subset X$. In particular, $\operatorname{En}(T) \subset X$. It follows that $X$ is not connected, because otherwise $X=T$, a contradiction.

Since $f$ maps any connected component of $X$ onto a connected component of $X$ and $P$ is a periodic orbit of $f$, it easily follows that $f$ acts as a cyclic permutation of the set of connected components of $X$. So, there exists a divisor $p \geq 2$ of $n$ 
and a labelling $X_{1}, X_{2} \ldots, X_{p}$ of the set of connected components of $X$ such that $f\left(X_{i}\right)=X_{i+1}$ for $1 \leq i<p$ and $f\left(X_{p}\right)=X_{1}$. Set $P_{i}=X_{i} \cap P$ for $1 \leq i \leq p$. Then, $f\left(P_{i}\right)=P_{i+1}$ for $1 \leq i<p$ and $f\left(P_{p}\right)=P_{1}$. Moreover, for any pair $\{i, j\} \subset\{1,2, \ldots, p\}$ we have that $\left\langle P_{i}\right\rangle \cap\left\langle P_{j}\right\rangle=\emptyset$, since $X_{i} \cap X_{j}=\emptyset$. Thus, $\mathcal{P}$ has a separated $p$-block structure and the proof of $(\mathrm{a})$ is now complete.

Let us prove (b). We start by proving the "if" part of the statement. If $\mathcal{P}$ is a trivial pattern, Remark 1.3 tells us that $M$ is a cyclic matrix. On the other hand, if $\mathcal{P}$ is non-trivial and there is a partition $P=P_{1} \cup P_{2} \cup \ldots \cup P_{p}$ defining a $p$-rotational structure for $\mathcal{P}$, then Lemma 3.2 tells us as $M$ reads as the right hand side of (2). Hence, $M$ is cyclic.

Finally we prove the "only if" part of (b). Until the end of this proof, for any $k \in \mathbb{N}$ we will take $\{1,2, \ldots, k\}$ as the representatives of $\mathbb{Z} / k$. Let $I_{1}, I_{2}, \ldots, I_{z}$ be a labelling of the set of $P_{V}$-basic intervals such that, for some $p \geq 2$,

$$
M=\left(\begin{array}{ccccc}
0 & M_{1} & 0 & \ldots & 0 \\
0 & 0 & M_{2} & & 0 \\
\vdots & & \ddots & \ddots & \vdots \\
0 & 0 & \ldots & 0 & M_{p-1} \\
M_{p} & 0 & \ldots & 0 & 0
\end{array}\right)
$$

where the diagonal 0 blocks are square. Assume that $\mathcal{P}$ is not trivial. We have to show that $\mathcal{P}$ has a rotational structure.

Let $m_{i}$ be the number of rows of $M_{i}$ for $1 \leq i \leq p$, so that $m_{1}+m_{2}+\ldots+m_{p}=z$. Set $X_{1}=\bigcup_{j=1}^{m_{1}} I_{j}$ and $X_{i}=\bigcup_{j=m_{1}+\ldots+m_{i-1}+1}^{m_{1}+\ldots+m_{j}} I_{j}$ for $2 \leq i \leq p$. Since $M$ has the above cyclic form,

$$
f\left(X_{i}\right) \subset X_{i+1 \bmod p} \text { for } 1 \leq i \leq p .
$$

Observe that any $P_{V}$-basic interval belongs to a unique $X_{i}$. Moreover, if a $P_{V}$ basic interval $I$ is contained in $X_{i}$ then $f(I)$ is either a point of $X_{i+1}$ or a connected union of $P_{V}$-basic intervals contained in $X_{i+1}$.

Take a fixed point $y$ of $f$. Note that $y \notin P$ since $P$ is an $n$-periodic orbit with $n>1$. Moreover, $y \in V(T)$ because if $y \in \operatorname{Int}(I)$ for some $P_{V}$-basic interval $I$ then $f(I) \supset I$, in contradiction with the fact that all the diagonal entries of $M$ are 0 . Let $s \geq 3$ be the valence of $y$. Let $\left\{a_{i}\right\}_{i=1}^{s}$ be the set of points from $P_{V}$ such that $\left(y, a_{i}\right) \cap P_{V}=\emptyset$ for $1 \leq i \leq s$. Now we claim that $f\left(a_{i}\right) \neq y$. Indeed: this is obvious when $a_{i} \in P$ and, if $a_{i} \in V(T) \backslash P$, then $f\left(a_{i}\right) \neq y$ because otherwise $\left[y, a_{i}\right]$ would be an invariant forest, in contradiction with the fact that $(T, P, f)$ is a canonical model.

Now observe that each $\left[y, a_{i}\right]$ is a $P_{V}$-basic interval and, in consequence, is contained in a unique $X_{j}$. From the previous claim and (7), it immediately follows that $s=p \cdot r$ for some $r \geq 1$ and $f$ acts as a local rotation around $y$. Without loss of generality we may assume that the points $a_{i}$ are labelled in such a way that

$$
\left[y, a_{i}\right] \subset X_{i \bmod p} \text { for } 1 \leq i \leq s .
$$

Let $Z_{1}, Z_{2}, \ldots, Z_{s}$ be a labelling of the set of connected components of $T \backslash\{y\}$ such that $a_{i} \in Z_{i}$ for $1 \leq i \leq s$. Set $Y_{i}:=\mathrm{Cl}\left(\bigcup_{j=0}^{r-1} Z_{i+j p}\right)$, for $1 \leq i \leq p$. We claim that $Y_{i}=X_{i}$ for $i=1,2, \ldots, p$.

Now we prove the claim. First we will show that $[y, w] \subset X_{i}$ for every $w \in$ $\operatorname{En}(T) \cap Y_{i}$.

Recall that $(T, P, f)$ has no invariant forests. Then, from the fact that $f(y)=y$ and the $P$-monotonicity of $f$ it follows that there exists $t \geq 0$ such that $f^{i}\left(\left[y, a_{1}\right]\right) \cap$ $P=\emptyset$ for $0 \leq i<t, f^{t}\left(\left[y, a_{1}\right]\right) \cap P \neq \emptyset$ and $f^{i}\left(\left[y, a_{1}\right]\right)=\left[y, f^{i}\left(a_{1}\right)\right] \supset\left[y, a_{1+i \bmod s}\right]$ 
for $0 \leq i \leq t$. Take any $x \in\left[y, f^{t}\left(a_{1}\right)\right] \cap P$. Then, $[y, x] \supset\left[y, a_{1+t} \bmod s\right]$. By (8), $\left[y, a_{1}\right] \subset X_{1}$ and, by $(7)$,

$$
[y, x] \subset X_{1+t \bmod p} .
$$

Now, since $\operatorname{En}(T) \subset P$, it follows that $w \in P$. So, there is $r \geq 0$ such that $w=f^{r}(x)$. From (9) and using again (7), we have that

$$
X_{1+t+r} \bmod p \supset f^{r}([y, x]) \supset\left[y, f^{r}(x)\right]=[y, w] .
$$

On the other hand, there exists a unique $j \in\{1,2, \ldots, s\}$ such that $[y, w] \supset\left[y, a_{j}\right]$. By the definition of the sets $Y_{i}$ and the assumption that $w \in Y_{i}$, we get $j \equiv i$ $(\bmod p)$. On the other hand, from $(8)$ and $(10), j \equiv 1+t+r(\bmod p)$. In consequence, $X_{1+t+r \bmod p}=X_{i}$. Therefore, $Y_{i}=\bigcup_{w \in \operatorname{En}(T) \cap Y_{i}}[y, w] \subset X_{i}$.

Now assume that $Y_{i} \subsetneq X_{i}$. Since both sets are unions of $P_{V}$-basic intervals it follows that $X_{i} \backslash Y_{i}$ contains the interior of a $P_{V}$-basic interval $J$. Since $T=\bigcup_{i=1}^{p} Y_{i}$ it follows that $J \subset Y_{j} \subset X_{j}$ with $j \neq i$. Consequently the $P_{V^{-}}$-basic interval $J$ is contained simultaneously in $X_{i}$ and $X_{j}$; a contradiction. This ends the proof of the claim.

From (7) it follows that, for each $i=1,2, \ldots, p, f\left(Y_{i}\right) \subset Y_{i+1} \bmod p$. We have to see that $f\left(Y_{i}\right)=Y_{i+1} \bmod p$ for every $i=1,2, \ldots, p$.

Set $P_{i}:=P \cap Y_{i}$. Since $T=\bigcup_{i=1}^{p} Y_{i}$ and $P$ is a periodic orbit it follows that $\left|P_{i}\right|=n / p$ for every $i$ and $f\left(P_{i}\right)=P_{i+1} \bmod p$. To see that $f\left(Y_{i}\right)=Y_{i+1} \bmod p$ we will prove that $[y, w] \subset f\left(Y_{i}\right)$ for every $w \in \operatorname{En}(T) \cap Y_{i+1} \bmod p$. Since

$$
w \in \operatorname{En}(T) \cap Y_{i+1} \bmod p \subset P \cap Y_{i+1} \bmod p=P_{i+1} \bmod p,
$$

there exists a unique $x \in P_{i} \subset Y_{i}$ such that $f(x)=w$. Consequently, $[y, w] \subset$ $f([y, x]) \subset Y_{i+1} \bmod p$ because $[y, x] \subset Y_{i}$ and $f\left(Y_{i}\right) \subset Y_{i+1} \bmod p$. This implies that $f\left(Y_{i}\right)=Y_{i+1} \bmod p$ and, hence, $\mathcal{P}$ has a $p$-rotational structure.

Finally we prove Corollary $\mathrm{C}$, which follows easily from Corollary B and the definition of a simplicial model.

Proof of Corollary $C$. Let $(T, P, f)$ be the canonical model of $\mathcal{P}$. Since $\mathcal{P}$ is simplicial, $V(T) \subset P$. Since $n \geq 3$, it follows that $\mathcal{P}$ has at least two discrete components and, in consequence, is not a trivial pattern.

Recall that a collapsing interval has the form $[a, b]$ with $a \in P$ and $b \in V(T) \backslash$ $P$. Since $V(T) \subset P$, it follows that $\mathcal{P}$ has no collapsing intervals. Then, by Theorem A(a), $M$ is reducible if and only if $\mathcal{P}$ has a separated block structure.

On the other hand, the fact that $V(T) \subset P$ implies that the set of fixed points of $f$, that is disjoint from $P$, is disjoint from $V(T)$. It follows that $\mathcal{P}$ cannot have a rotational structure. Then, the corollary follows from Lemma 1.4 and Corollary B.

\section{Proof of Theorem D}

For any square matrix $M$, we will denote its spectral radius by $\sigma(M)$. We recall that it is defined as the maximum of the moduli of the eigenvalues of $M$. It is well known that the topological entropy of an interval pattern $\mathcal{P}$ can be computed as $\log \max \{\sigma, 1\}$, where $\sigma$ is the spectral radius of the Markov matrix of a monotone representative of $\mathcal{P}$. This is also true for general tree patterns. Indeed, let $\mathcal{P}$ be a pattern. Let $(T, P, f)$ be a monotone representative of $\mathcal{P}$. By Theorem 1.1(b), $h(\mathcal{P})=h(f)$. On the other hand, $f$ is $(P \cup V(T))$-monotone and thus we can consider the Markov matrix of the monotone model $(T, P \cup V(T), f)$. By standard arguments (see for instance [4, Theorem 4.4.5] or [6]), the topological entropy of $f$ is equal to

$$
h(\mathcal{P})=\log \max \{\sigma(M), 1\} .
$$


Now we are ready to prove Theorem D.

Proof of Theorem $D$. Set $P_{V}=P \cup V(T)$. Let $z$ be the number of $P_{V}$-basic intervals, let $n_{i} \geq 1$ be the number of $P_{V}$-basic intervals contained in $\left\langle P_{i}\right\rangle$ for $i=1,2, \ldots, p$ and let $q=n_{1}+n_{2}+\ldots+n_{p}$. Then, by Lemmas 3.1 and 3.2, there exist positive integers $m_{1}, m_{2}, \ldots, m_{p}$ and a labelling of the set of $P_{V}$-basic intervals such that the Markov matrix of $\mathcal{P}$ reads as

$$
\left(\begin{array}{cc}
M_{11} & 0 \\
M_{21} & M_{22}
\end{array}\right)
$$

with

$$
M_{11}=\left(\begin{array}{ccccc}
0 & M_{1} & 0 & \ldots & 0 \\
0 & 0 & M_{2} & & 0 \\
\vdots & & \ddots & \ddots & \vdots \\
0 & 0 & \ldots & 0 & M_{p-1} \\
M_{p} & 0 & \ldots & 0 & 0
\end{array}\right),
$$

where $M_{i}$ is an $m_{i} \times m_{i+1}$ matrix for $1 \leq i<p, M_{p}$ is an $m_{p} \times m_{1}$ matrix, and:

Case 1: If $P_{1} \cup P_{2} \cup \ldots \cup P_{p}$ defines a $p$-rotational structure for $\mathcal{P}$, then $M_{22}$ is an empty matrix and, for each $1 \leq i \leq p$, either $m_{i}=n_{i}+1$ when all blocks are pairwise disjoint, or $m_{i}=n_{i}$ when they all intersect at a single branching point.

Case 2: If $P_{1} \cup P_{2} \cup \ldots \cup P_{p}$ defines a separated $p$-block structure for $\mathcal{P}$ that is not a $p$-rotational structure, then $q<z$ and $m_{i}=n_{i}$ for each $1 \leq i \leq p$. Thus, $M_{11}$ is a $q \times q$ matrix and $M_{22}$ is a $(z-q) \times(z-q)$ matrix.

It is straightforward to check that

$$
\left(M_{11}\right)^{p}=\left(\begin{array}{cccc}
N_{1} & 0 & \ldots & 0 \\
0 & N_{2} & & \vdots \\
\vdots & & \ddots & 0 \\
0 & \ldots & 0 & N_{p}
\end{array}\right),
$$

where

(13) $\quad N_{1}=M_{1} M_{2} \cdots M_{p}$ and $N_{i}=M_{i} M_{i+1} \cdots M_{p} M_{1} \cdots M_{i-1}$ for $2 \leq i \leq p$.

So, $N_{i}$ is an $m_{i} \times m_{i}$ square matrix for each $1 \leq i \leq p$.

Next let us see that

$$
\sigma\left(N_{i}\right)=\sigma\left(N_{j}\right) \text { for each pair } i, j \in\{1,2, \ldots, p\} .
$$

Indeed, it is well known (see for instance [4, Lemma 4.4.2]) that, for every matrix $\mathcal{N}$,

$$
\sigma(\mathcal{N})=\limsup _{k \rightarrow \infty}\left|\operatorname{tr}\left(\mathcal{N}^{k}\right)\right|^{1 / k}
$$

where $\operatorname{tr}(\cdot)$ denotes the trace of a square matrix (that is, the sum of all the entries of its diagonal). Therefore, to prove (14) it is enough to show that $\operatorname{tr}\left(\left(N_{i}\right)^{k}\right)=$ $\operatorname{tr}\left(\left(N_{j}\right)^{k}\right)$ for all $k \geq 1$. Using (13) and the definition of the product of matrices, it immediately follows that, for every $1 \leq t \leq p$, the $m$-th entry of the diagonal of $\left(N_{t}\right)^{k}$ coincides with the number of different loops of length $k$ in the Markov graph of $\left(T, P_{V}, f\right)$ starting and ending at the corresponding $P_{V}$-basic interval in $\left\langle P_{t}\right\rangle$. Therefore, $\operatorname{tr}\left(\left(N_{t}\right)^{k}\right)$ is the total number of loops of length $k$ in the Markov graph of $\left(T, P_{V}, f\right)$ starting and ending at some $P_{V}$-basic interval in $\left\langle P_{t}\right\rangle$. Let us denote the set of all such loops as $\Gamma_{t}^{k}$.

Now we claim that for each $k \geq 1$ there exists a one-to-one correspondence $\phi: \Gamma_{i}^{k} \longrightarrow \Gamma_{j}^{k}$. Clearly, (14) follows from this claim and the previous paragraph. Let us prove the claim. Assume for definiteness that $j \geq i$. Let $\alpha \in \Gamma_{i}^{k}$. By 
Lemma 2.1, $f\left(\left\langle P_{t}\right\rangle\right)=\left\langle P_{t+1}\right\rangle$ for $1 \leq t<p$ and $f\left(\left\langle P_{p}\right\rangle\right)=\left\langle P_{1}\right\rangle$. It easily follows that $k$ is a multiple of $p$ and that $\alpha$ has the form

$$
I_{i} \rightarrow I_{i+1} \rightarrow \ldots \rightarrow I_{p} \rightarrow I_{p+1} \rightarrow I_{p+2} \rightarrow \ldots \rightarrow I_{i+k-1} \rightarrow I_{i},
$$

with $I_{t} \in\left\langle P_{t \bmod }\right\rangle$ for $i \leq t \leq i+k$ (we take $\{1,2, \ldots, p\}$ as the representatives of $\mathbb{N} / p)$. We define $\phi(\alpha)$ to be the shifted loop

$$
I_{j} \rightarrow I_{j+1} \rightarrow \ldots \rightarrow I_{p} \rightarrow I_{p+1} \rightarrow I_{p+2} \rightarrow \ldots \rightarrow I_{i+k-1} \rightarrow I_{i} \rightarrow I_{i+1} \rightarrow \ldots \rightarrow I_{j},
$$

which is a loop of length $k$ starting and ending at a $P_{V}$-basic interval in $\left\langle P_{j}\right\rangle$. So, $\phi(\alpha) \in \Gamma_{j}^{k}$. Clearly, $\phi$ is one-to-one and the claim is proved.

Now we will prove that

$$
h\left(\left.f^{p}\right|_{\left\langle P_{t}\right\rangle}\right)=\log \max \left\{\sigma\left(N_{t}\right), 1\right\} \quad \text { for every } 1 \leq t \leq p .
$$

Set $\widetilde{Q}:=P_{V} \cup \operatorname{Bd}\left(f^{-p}\left(P_{V}\right)\right)$. The fact that $(T, P, f)$ is a monotone model implies that $f^{-p}\left(P_{V}\right)$ has finitely many connected components. Hence, since $\operatorname{Bd}\left(f^{-p}\left(P_{V}\right)\right)$ is the union of $\operatorname{Bd}(C)$ for every connected component $C$ of $f^{-p}\left(P_{V}\right)$, it follows that $\widetilde{Q}$ is finite.

Now we set $Q_{t}:=\widetilde{Q} \cap\left\langle P_{t}\right\rangle$. Since $P_{V}$ is $f$-invariant it follows that

$$
P_{V} \subset f^{-1}\left(P_{V}\right) \subset f^{-2}\left(P_{V}\right) \subset \cdots \subset f^{-p}\left(P_{V}\right) .
$$

Hence, $\widetilde{Q} \subset f^{-p}\left(P_{V}\right)$ and, consequently,

$$
f^{p}\left(Q_{t}\right) \subset f^{p}\left(f^{-p}\left(P_{V}\right) \cap\left\langle P_{t}\right\rangle\right) \subset P_{V} \cap\left\langle P_{t}\right\rangle \subset Q_{t}
$$

because $f^{p}\left(\langle P\rangle_{t}\right) \subset\left\langle P_{t}\right\rangle$. So, $Q_{t}$ is $\left.f^{p}\right|_{\left\langle P_{t}\right\rangle}$-invariant and $V(T) \cap\left\langle P_{t}\right\rangle \subset \widetilde{Q} \cap\left\langle P_{t}\right\rangle=$ $Q_{t}$. Hence, we can speak about $Q_{t}$-basic intervals (in $\left\langle P_{t}\right\rangle$ ) and consider the model $\left(\left\langle P_{t}\right\rangle, Q_{t},\left.f^{p}\right|_{\left\langle P_{t}\right\rangle}\right)$.

The connected components of $T \backslash \widetilde{Q}$ (and hence of $\left\langle P_{t}\right\rangle \backslash Q_{t}$ ) are characterized by the following claim: every connected component of $T \backslash \widetilde{Q}$ is either a connected component of $T \backslash f^{-p}\left(P_{V}\right)$ or its closure is contained in (a connected component of) $f^{-p}\left(P_{V}\right)$. To see it, let $C$ be a connected component of $T \backslash \widetilde{Q}$. Clearly, either $C$ is a connected component of $T \backslash f^{-p}\left(P_{V}\right)$ or $C \cap f^{-p}\left(P_{V}\right) \neq \emptyset$. Since $V(T) \cup \operatorname{Bd}\left(f^{-p}\left(P_{V}\right)\right) \subset \widetilde{Q}$, it follows that $C$ is an interval disjoint from $V(T)$ and $\mathrm{Bd}\left(f^{-p}\left(P_{V}\right)\right)$. Hence, $C \cap f^{-p}\left(P_{V}\right) \neq \emptyset$ implies that $\mathrm{Cl}(C)$ is contained in a connected component of $f^{-p}\left(P_{V}\right)$. This proves the claim.

Now we claim that $\left.f^{p}\right|_{J}$ is monotone for every $Q_{t}$-basic interval $J$. Clearly, the $f^{p}$-image of every connected component of $f^{-p}\left(P_{V}\right)$ is a single point. Hence, the claim holds trivially when $J$ is contained in a connected component of $f^{-p}\left(P_{V}\right)$. If this is not the case, by the above claim, $J$ is the closure of a connected component of $T \backslash f^{-p}\left(P_{V}\right)$. To prove that $\left.f^{p}\right|_{J}$ is monotone it is enough to show that $f^{i}(J)$ is contained in a $P_{V}$-basic interval for $i=0,1, \ldots, p$, because the model $\left(T, P_{V}, f\right)$ is monotone and

$$
\left.f^{p}\right|_{J}=\left.\left.\left.\left.f\right|_{f^{p-1}(J)} \circ f\right|_{f^{p-2}(J)} \circ \cdots \circ f\right|_{f(J)} \circ f\right|_{J} .
$$

We have $\emptyset=\operatorname{Int}(J) \cap f^{-p}\left(P_{V}\right) \supset \operatorname{Int}(J) \cap f^{-i}\left(P_{V}\right)$ for every $i \in\{0,1, \ldots, p\}$. Hence, $f^{i}(J)$ is contained in a $P_{V}$-basic interval for $i=0,1, \ldots, p$. This ends the proof of the monotonicity of $\left.f^{p}\right|_{J}$.

Let $B$ denote the Markov matrix of the monotone model $\left(\left\langle P_{t}\right\rangle, Q_{t},\left.f^{p}\right|_{\left\langle P_{t}\right\rangle}\right)$. Then, by (11), $h\left(\left.f^{p}\right|_{\left\langle P_{t}\right\rangle}\right)=\log \max \{\sigma(B), 1\}$. Thus, to prove (15), we have to show that $\sigma(B)=\sigma\left(N_{t}\right)$. It is well known (see, for instance, [11]) that the spectral radius of a non-negative matrix $\mathcal{N}$ is equal to the limit as $k$ goes to infinity of the $k$-th root of the norm of $\mathcal{N}^{k}$, where we can use any norm equivalent to the standard 
one. In particular we can use the 1-entrywise matrix norm, which we will denote by $\|\cdot\|_{1}$ and is defined for non-negative matrices as the sum of all entries. Then,

$$
\sigma(\mathcal{N})=\lim _{k \rightarrow \infty} \sqrt[k]{\left\|\mathcal{N}^{k}\right\|_{1}}
$$

So we will have to study and relate the entries of $B^{k}$ and $N_{t}^{k}$ for every $k$. In particular we have to fix the labelling of the $Q_{t}$-basic intervals.

Let $I_{\widetilde{m}_{t}+1}, I_{\widetilde{m}_{t}+2}, \ldots, I_{\widetilde{m}_{t}+m_{t}}$ denote the $P_{V}$-basic intervals contained in $\left\langle P_{t}\right\rangle$ (labelled as in the definition of the matrix $M$ ), where $\widetilde{m}_{t}=\sum_{i=0}^{t-1} m_{i}$.

Since $P_{V} \cap\left\langle P_{t}\right\rangle \subset Q_{t}$, it follows that every $Q_{t}$-basic interval is contained in one of the intervals $I_{\widetilde{m}_{t}+\ell}$. Then, for $\ell \in\left\{1,2, \ldots, m_{t}\right\}$ let us denote by $r_{\ell}$ the number of $Q_{t}$-basic intervals contained in $I_{\widetilde{m}_{t}+\ell}$ and label these intervals by $J_{\ell, i}$ with $i \in$ $\left\{1,2, \ldots, r_{\ell}\right\}$. For $\ell, m \in\left\{1,2, \ldots, m_{t}\right\}$ we define a matrix $B_{\ell, m}=B_{\ell, m}^{(1)}=\left(b_{i, j}^{\ell, m}\right)$ of size $r_{\ell} \times r_{m}$ by

$$
b_{i, j}^{\ell, m}= \begin{cases}1 & \text { if } J_{\ell, i} f^{p} \text {-covers } J_{m, j} \\ 0 & \text { otherwise. }\end{cases}
$$

Observe that, with the above labelling of the $Q_{t}$-basic intervals, the matrix $B$ can be written in block form as

$$
B=\left(\begin{array}{cccc}
B_{1,1} & B_{1,2} & \cdots & B_{1, m_{t}} \\
B_{2,1} & B_{2,2} & \cdots & B_{2, m_{t}} \\
\vdots & \vdots & \cdots & \vdots \\
B_{m_{t}, 1} & B_{m_{t}, 2} & \cdots & B_{m_{t}, m_{t}}
\end{array}\right)
$$

Also, for $k \geq 1$ we set

$$
B_{\ell, m}^{(k+1)}:=\sum_{s=1}^{m_{t}} B_{\ell, s}^{(k)} B_{s, m}
$$

for every $\ell, m$. Then, by standard Algebra, $B^{k}$ is the block matrix $\left(B_{\ell, m}^{(k)}\right)$.

By construction of the set $Q_{t}$ we have $f^{p}\left(Q_{t}\right) \subset P_{V} \cap\left\langle P_{t}\right\rangle$. This means that, given $\ell, m \in\left\{1,2, \ldots, m_{t}\right\}$ and $i \in\left\{1,2, \ldots, r_{\ell}\right\}$, either $J_{\ell, i} f^{p}$-covers $I_{\widetilde{m}_{t}+m}$ or $f^{p}\left(J_{\ell, i}\right)$ is disjoint from $\operatorname{Int}\left(I_{\widetilde{m}_{t}+m}\right)$. In other words, the $i$-th row of $B_{\ell, m}$ is either constant 0 or constant 1 . In particular, every matrix $B_{\ell, m}$ is row-constant according to the following definition.

A non-negative matrix such that, for every row, all entries in that row coincide will be called row-constant. For operational purposes, we will write a row-constant matrix of size $s_{1} \times s_{2}$ as $v u_{s_{2}}^{T}$ where $v$ is a non-negative vector of size $s_{1}, u_{s_{2}}$ denotes a vector of ones of size $s_{2}$ and $u_{s_{2}}^{T}$ means the transpose of $u_{s_{2}}$ (here "vector" means a matrix with a single column, and thus $u_{s_{2}}^{T}$ is a matrix with a single row). Observe that each column of a row-constant matrix coincides with the vector $v$ and hence its sum is $\|v\|_{1}$ (which, by definition, is the sum of all entries of $v$ ).

Clearly the sum of row-constant matrices is row-constant. On the other hand, if $v$ is a non-negative vector of size $s_{1}, w$ is a non-negative vector of size $s_{2}$ and $s_{3}$ is a positive integer, then $\left(v u_{s_{2}}^{T}\right)\left(w u_{s_{3}}^{T}\right)=v\left(u_{s_{2}}^{T} w\right) u_{s_{3}}^{T}=\left(\|w\|_{1} v\right) u_{s_{3}}^{T}$. Hence, the product of row-constant matrices is also row-constant. Consequently, by (16), we obtain by induction on $k$ that every matrix $B_{\ell, m}^{(k+1)}$ is row-constant. 
We will denote by $\beta_{\ell, m}^{(k)}$ the non-negative integer vector of size $r_{\ell}$ such that $B_{\ell, m}^{(k)}=\beta_{\ell, m}^{(k)} u_{r_{m}}^{T}$. We also set $\beta_{\ell, m}=\beta_{\ell, m}^{(1)}$. Hence, equation (16) now becomes

$$
\begin{aligned}
B_{\ell, m}^{(k+1)} & =\sum_{s=1}^{m_{t}} B_{\ell, s}^{(k)} B_{s, m}=\sum_{s=1}^{m_{t}}\left(\beta_{\ell, s}^{(k)} u_{r_{s}}^{T}\right)\left(\beta_{s, m} u_{r_{m}}^{T}\right)=\sum_{s=1}^{m_{t}} \beta_{\ell, s}^{(k)}\left(u_{r_{s}}^{T} \beta_{s, m}\right) u_{r_{m}}^{T} \\
& =\sum_{s=1}^{m_{t}}\left\|\beta_{s, m}\right\|_{1} \beta_{\ell, s}^{(k)} u_{r_{m}}^{T}=\left(\sum_{s=1}^{m_{t}}\left\|\beta_{s, m}\right\|_{1} \beta_{\ell, s}^{(k)}\right) u_{r_{m}}^{T},
\end{aligned}
$$

and consequently,

$$
\beta_{\ell, m}^{(k+1)}=\sum_{s=1}^{m_{t}}\left\|\beta_{s, m}\right\|_{1} \beta_{\ell, s}^{(k)}
$$

Now we set $N_{t}:=\left(a_{\ell, m}\right)$ and, for every $k \geq 1, N_{t}^{k}=\left(a_{\ell, m}^{(k)}\right)$. We will prove by induction on $k$ that $a_{\ell, m}^{(k)}=\left\|\beta_{\ell, m}^{(k)}\right\|_{1}$ for every $k, \ell, m$.

We first consider the case $k=1$. As it has been noted above, $a_{\ell, m}$ coincides with the number of different paths of length $p$ in the Markov graph of $\left(T, P_{V}, f\right)$ from $I_{\widetilde{m}_{t}+\ell}$ to $I_{\widetilde{m}_{t}+m}$.

We start by proving that $a_{\ell, m} \leq\left\|\beta_{\ell, m}\right\|_{1}$. By [4, Lemma 1.2.6] every path of length $p$ in the Markov graph of $\left(T, P_{V}, f\right)$ from $I_{\widetilde{m}_{t}+\ell}$ to $I_{\widetilde{m}_{t}+m}$ gives a minimal closed subinterval $\widehat{J}$ of $I_{\widetilde{m}_{t}+\ell}$ such that $f^{s}(\widehat{J})$ is contained in a $P_{V}$-basic interval for $s=1,2, \ldots, p-1$ and $f^{p}(\widehat{J})=I_{\widetilde{m}_{t}+m}$. Then, the endpoints of $\widehat{J}$ belong to $f^{-p}\left(P_{V}\right) \cap\left\langle P_{t}\right\rangle$ and $\widehat{J}$ is the closure of a connected component of $\left\langle P_{t}\right\rangle \backslash f^{-p}\left(P_{V}\right)$. Hence, $\widehat{J}$ is a $Q_{t}$-basic interval $J_{\ell, i}$. This implies that the $i$-th entry of $\beta_{\ell, m}$ is 1. Moreover, two different paths of length $p$ in the Markov graph of $\left(T, P_{V}, f\right)$ from $I_{\widetilde{m}_{t}+\ell}$ to $I_{\widetilde{m}_{t}+m}$ give rise to two different $Q_{t}$-basic intervals. Consequently, $a_{\ell, m} \leq\left\|\beta_{\ell, m}\right\|_{1}$.

Now we will prove the converse inequality. Let $J_{\ell, i}$ be a $Q_{t}$-basic interval such that $J_{\ell, i} f^{p}$-covers $I_{\widetilde{m}_{t}+m}$ (i.e. the $i$-th entry of $\beta_{\ell, m}$ is 1$)$. Clearly $f^{p}\left(J_{\ell, i}\right)$ is non-degenerate and, hence, $J_{\ell, i}$ is the closure of a connected component of $\left\langle P_{t}\right\rangle \backslash$ $f^{-p}\left(P_{V}\right)$. From above, $f^{s}\left(J_{\ell, i}\right)$ is contained in a $P_{V}$-basic interval for $s=0,1, \ldots, p$ (in particular $\left.f^{p}\left(J_{\ell, i}\right)=I_{\widetilde{m}_{t}+m}\right)$. In a similar way to the proof of [4, Lemma 1.2.12], these $P_{V}$-basic intervals form a path of length $p$ in the Markov graph of $\left(T, P_{V}, f\right)$ from $I_{\widetilde{m}_{t}+\ell}$ to $I_{\widetilde{m}_{t}+m}$ and two different $Q_{t}$-basic intervals give rise to two different paths. Hence, $\left\|\beta_{\ell, m}\right\|_{1} \leq a_{\ell, m}$ and, consequently, $a_{\ell, m}^{(1)}=a_{\ell, m}=\left\|\beta_{\ell, m}\right\|_{1}=\left\|\beta_{\ell, m}^{(1)}\right\|_{1}$ for every $\ell, m$.

Now assume that $a_{\ell, m}^{(k)}=\left\|\beta_{\ell, m}^{(k)}\right\|_{1}$ holds for some $k \geq 1$ and for every $\ell, m$. Then, by $(17)$, the norm properties and the definition of the matrix product,

$$
\left\|\beta_{\ell, m}^{(k+1)}\right\|_{1}=\left\|\sum_{s=1}^{m_{t}}\right\| \beta_{s, m}\left\|_{1} \beta_{\ell, s}^{(k)}\right\|_{1}=\sum_{s=1}^{m_{t}}\left\|\beta_{\ell, s}^{(k)}\right\|_{1}\left\|\beta_{s, m}\right\|_{1}=\sum_{s=1}^{m_{t}} a_{\ell, s}^{(k)} a_{s, m}=a_{\ell, m}^{(k+1)}
$$

(notice that if $v$ and $w$ are non-negative vectors then $\|v+w\|_{1}=\|v\|_{1}+\|w\|_{1}$ ).

To end the proof of $(15)$, observe that $\left\|B_{\ell, m}^{(k)}\right\|_{1}$, the sum of all entries of $B_{\ell, m}^{(k)}$, is $r_{m}\left\|\beta_{\ell, m}^{(k)}\right\|_{1}$. Hence,

$$
\left\|B^{k}\right\|_{1}=\sum_{\ell, m=1}^{m_{t}}\left\|B_{\ell, m}^{(k)}\right\|_{1}=\sum_{\ell, m=1}^{m_{t}} r_{m}\left\|\beta_{\ell, m}^{(k)}\right\|_{1}=\sum_{\ell, m=1}^{m_{t}} r_{m} a_{\ell, m}^{(k)} .
$$


Consequently,

$$
\left\|N_{t}^{k}\right\|_{1} \leq\left\|B^{k}\right\|_{1} \leq r^{*}\left\|N_{t}^{k}\right\|_{1}
$$

where $r^{*}=\max _{m} r_{m}$. Thus,

$$
\sigma\left(N_{t}\right)=\lim _{k \rightarrow \infty} \sqrt[k]{\left\|N_{t}^{k}\right\|_{1}} \leq \lim _{k \rightarrow \infty} \sqrt[k]{\left\|B^{k}\right\|_{1}}=\sigma(B) \leq \lim _{k \rightarrow \infty} \sqrt[k]{r^{*}\left\|N_{t}^{k}\right\|_{1}}=\sigma\left(N_{t}\right) .
$$

This ends the proof of (15).

From (14) and (15) it follows that the entropies of $\left.f^{p}\right|_{\left\langle P_{i}\right\rangle}$ are all the same. On the other hand, using (11) and standard Algebra we get that

$$
\begin{aligned}
h(\mathcal{P})= & \log \max \left\{\sigma\left(M_{11}\right), \sigma\left(M_{22}\right), 1\right\}= \\
& \log \max \left\{\sigma\left(\left(M_{11}\right)^{p}\right)^{1 / p}, \sigma\left(M_{22}\right), 1\right\}= \\
& \log \max \left\{\sigma\left(N_{1}\right)^{1 / p}, \sigma\left(N_{2}\right)^{1 / p}, \ldots, \sigma\left(N_{p}\right)^{1 / p}, \sigma\left(M_{22}\right), 1\right\},
\end{aligned}
$$

where in this formula we formally set $\sigma\left(M_{22}\right)=1$ if $M_{22}$ is an empty matrix.

In view of (14), (15) and (18), to prove the theorem it is enough to show that

$$
h(\mathcal{Q})=\log \max \left\{\sigma\left(M_{22}\right), 1\right\},
$$

where $\mathcal{Q}$ is the skeleton of $\mathcal{P}$ and we formally set $\sigma\left(M_{22}\right)=1$ if $M_{22}$ is an empty matrix. We recall that $\mathcal{Q}=([S, Q],[\theta])$ with $Q=\left\{x_{1}, x_{2}, \ldots, x_{p}\right\}, \theta\left(x_{i}\right)=x_{i+1}$ for $1 \leq i<p$ and $\theta\left(x_{p}\right)=x_{1}$, and that the discrete components of $(S, Q)$ are defined according to the Cases 1 and 2 . Next we prove (19) in each case.

Case 1. $P=P_{1} \cup P_{2} \cup \ldots \cup P_{p}$ defines a $p$-rotational structure for $\mathcal{P}$.

Then, $M_{22}$ is an empty matrix and the right hand side of (19) is equal to 0 . Since in this case $\mathcal{Q}$ is by definition a trivial pattern, $h(\mathcal{Q})=0$. Thus, (19) holds and the theorem is proved in this case.

Case 2. $P=P_{1} \cup P_{2} \cup \ldots \cup P_{p}$ defines a separated $p$-block structure for $\mathcal{P}$ that is not a p-rotational structure.

Then, $q<z$ and $M_{22}$ is a $(z-q) \times(z-q)$ matrix. Now we recover the notation of the definition of the skeleton (page 9, case (b)). To compute the topological entropy of the skeleton $\mathcal{Q}$, next we construct a monotone model $(S, Q, g)$ of $\mathcal{Q}$. Let $g: S \longrightarrow S$ be such that $g \circ \kappa=\kappa \circ f$. Then, by the definition of $\kappa, g$ is well defined and $(S, Q, g)$ is a model of $\mathcal{Q}$. We claim that $g$ is $Q$-monotone. Observe that $\kappa$ is monotone on each interval $[a, b] \subset T$. Take any basic path $\sigma$ of $(S, Q)$. We have to see that $g$ is monotone on $\langle\sigma\rangle_{S}$. Note that there exists a basic path $\pi$ of $(T, P)$ such that $\kappa\left(\langle\pi\rangle_{T}\right)=\langle\sigma\rangle_{S}$. By the definitions, $\left.g\right|_{\langle\sigma\rangle_{S}}=\left.\kappa \circ f\right|_{\langle\pi\rangle_{T}}$, which is monotone. This ends the proof of the claim.

Set $Q_{V}:=Q \cup V(S)$. Let $\mathcal{A}$ be the set of $P_{V}$-basic intervals whose interior is contained in $T \backslash\left(\bigcup_{i=1}^{p}\left\langle P_{i}\right\rangle\right)$ and let $\mathcal{B}$ be the set of all $Q_{V}$-basic intervals. From the definitions it easily follows that if $v \in V(T)$ then $\kappa(v) \in V(S) \cup Q$, and that if $[v, w] \in \mathcal{A}$ then $[\kappa(v), \kappa(w)] \in \mathcal{B}$. Moreover, the correspondence $\widetilde{\kappa}$ which sends each $[v, w] \in \mathcal{A}$ to $[\kappa(v), \kappa(w)] \in \mathcal{B}$ is a bijection. On the other hand, the definition of $g$ implies that for any $I, J \in \mathcal{A}, I f$-covers $J$ if and only if $\widetilde{\kappa}(I) g$-covers $\widetilde{\kappa}(J)$. This means that if $I_{1}, I_{2}, \ldots, I_{z-q}$ is the labelling of $\mathcal{A}$ such that the Markov matrix of $\left(T, P_{V}, f\right)$ reads as in (12), then the Markov matrix of $\left(S, Q_{V}, g\right)$ associated to the labelling $\widetilde{\kappa}\left(I_{1}\right), \widetilde{\kappa}\left(I_{2}\right), \ldots, \widetilde{\kappa}\left(I_{z-q}\right)$ is exactly $M_{22}$. Since $(S, Q, g)$ is a monotone model of $\mathcal{Q},(11)$ tells us that (19) holds. 


\section{Proofs of Theorems E And F}

We start this section recalling the characterization of zero entropy patterns given in [3]. We will use this characterization to prove Theorems $\mathrm{E}$ and $\mathrm{F}$.

Let $(T, P, f)$ be a monotone model of a pattern $\mathcal{P}$. Let $\pi$ be a basic path of $(T, P)$. We say that $\mathcal{P}$ is $\pi$-reducible if $f^{n}(\pi)$ is contained in a single discrete component of $(T, P)$ for every $n \geq 0$. In this case, let $X=\bigcup_{i>0}\left\langle f^{i}(\pi)\right\rangle$ and let $C_{1}, C_{2}, \ldots, C_{p}$ be the connected components of $X$. Note that $P \subset X$. It is easy to see that for each $1 \leq i \leq p$ there exists $j_{i}$ such that $f\left(C_{i}\right) \subset C_{j_{i}}$. Then we take the tree $T^{\prime}$ obtained from $T$ by collapsing each $C_{i}$ to a point $c_{i}$. Let $\kappa: T \longrightarrow T^{\prime}$ be the standard projection. We set $P^{\prime}=\kappa(P)$ and define $f^{\prime}: P^{\prime} \longrightarrow P^{\prime}$ as $f^{\prime}=\kappa \circ f \circ \kappa^{-1}$. It is easy to see that $\left(\left[T^{\prime}, P^{\prime}\right],\left[f^{\prime}\right]\right)$ is a well defined pattern, which we call a $\pi$-reduced (or simply reduced) pattern of $\mathcal{P}$. The process of obtaining this pattern from $\mathcal{P}$ is called a reduction. A pattern will be called strongly reducible if there exists a finite sequence or reductions leading to a pattern $([S, Q],[g])$ such that $Q$ consists on a single point. The notion of a strongly reducible pattern depends apparently on the chosen sequence of basic paths and monotone models. From the next theorem, which is the characterization of zero entropy patterns given in [3], it follows that this notion is well defined.

Theorem 5.1 (Theorem E of [3]). A pattern has zero entropy if and only if it is strongly reducible.

The following result will be used to prove the "only if" part of the statement of Theorem E.

Proposition 5.2. Let $(T, P, f)$ be the canonical model of a periodic pattern $\mathcal{P}$ which is $\pi$-reducible for a basic path $\pi$ of $(T, P)$. Let $C$ be a connected component of $\bigcup_{i \geq 0}\left\langle f^{i}(\pi)\right\rangle$. Then, the pointed tree $(C, P \cap C)$ has a unique discrete component.

Proof. Set $\pi=\{a, b\}$ and $X=\bigcup_{i \geq 0}\left\langle f^{i}(\pi)\right\rangle$. By definition, $f^{i}(\pi)$ is a basic path of $(T, P)$ for $i \geq 0$. Since $f$ is $P$-monotone, it follows that $\left\langle f^{i}(\pi)\right\rangle=f^{i}(\langle\pi\rangle)$ and

$$
\left(f^{i}(a), f^{i}(b)\right) \cap P=\emptyset \text { for every } i \geq 0 .
$$

By the definition of the set $X$, every point $x \in P \cap C$ satisfies that:

(a) $x=f^{i}(a)$ for some $i \geq 0$ and $\left[x, f^{i}(b)\right] \subset C$

(b) $x=f^{j}(b)$ for some $j \geq 0$ and $\left[x, f^{j}(a)\right] \subset C$.

Since there exist only two integers $i, j \geq 0$ such that $x=f^{i}(a)$ and $x=f^{j}(b)$, it follows that:

(c) Either $\left[x, f^{i}(b)\right] \cap\left[x, f^{j}(a)\right]=\{x\}$ and, thus, $\operatorname{Val}_{C}(x)=2$, or $\left[x, f^{i}(b)\right] \cap$ $\left[x, f^{j}(a)\right]$ has non-empty interior and, thus, $\operatorname{Val}_{C}(x)=1$.

For simplicity, by shifting the labels of the points of $P$ we can assume without loss of generality that $a \in C$.

Since $f$ is $P$-monotone, it follows that the set $X$ is $f$-invariant. Let $p$ be the number of connected components of $X$. Set $g=f^{p}$ and $r=|P| / p$. Since $P$ is a periodic orbit, $f$ acts as a $p$-cyclic permutation of the set of connected components of $X$ and, in consequence, $P \cap C$ is an $r$-periodic orbit of $g$. Using (a-c), this fact implies that every point $x \in P \cap C$ satisfies:

(A) $x=g^{i}(a)$ for some $i \geq 0$ and $\left[x, g^{i}(b)\right] \subset C$

(B) $x=g^{j}(b)$ for some $j \geq 0$ and $\left[x, g^{j}(a)\right] \subset C$

(C) Either $\left[x, g^{i}(b)\right] \cap\left[x, g^{j}(a)\right]=\{x\}$ and, thus, $\operatorname{Val}_{C}(x)=2$, or $\left[x, g^{i}(b)\right] \cap\left[x, g^{j}(a)\right]$ has non-empty interior and, thus, $\operatorname{Val}_{C}(x)=1$.

To prove the proposition, it is enough to show that $\operatorname{Val}_{C}(x)=1$ for each $x \in$ $P \cap C$. For definiteness, by shifting again the labels of the points of $P$ if necessary, 


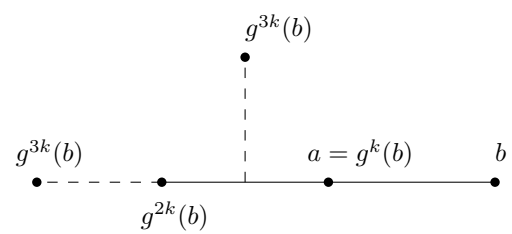

Figure 7 . The two possible relative positions of the point $g^{3 k}(b)$ in the proof of Proposition 5.2, when $g^{3 k}(b) \neq b$.

we may assume that $x=a$. Let $k \geq 1$ be such that $g^{k}(b)=a$. Since obviously (20) holds with $g$ instead of $f$, it follows that

$$
\left(g^{i+k}(b), g^{i}(b)\right) \cap P=\emptyset \text { for every } i \geq 0 .
$$

Moreover, using (A-C) with $i=0, j=k$ and $x=a=g^{k}(b)$ we get that either $\left[g^{k}(b), b\right] \cap\left[g^{k}(b), g^{2 k}(b)\right]=\left\{g^{k}(b)\right\}$ and $\operatorname{Val}_{C}(a)=\operatorname{Val}\left(g^{k}(b)\right)=2$ or $\left[g^{k}(b), b\right] \cap$ $\left[g^{k}(b), g^{2 k}(b)\right]$ has non-empty interior and $\operatorname{Val}_{C}(a)=\operatorname{Val}\left(g^{k}(b)\right)=1$ (see Figure 7 for a sketch of this situation). Next we will show that the first possibility does not hold. To do it, assume by way of contradiction that $b$ and $g^{2 k}(b)$ are separated by $g^{k}(b)$.

If $g^{3 k}(b)=b$ then $g^{k}(b) \in\left(g^{2 k}(b), g^{3 k}(b)\right) \cap P=\left(g^{2 k}(b), b\right) \cap P$, in contradiction with (21). On the other hand, if $g^{3 k}(b) \neq b$, we use (A-C) with $i=k$ and $j=2 k$ to conclude that either $\operatorname{Val}_{C}\left(g^{2 k}(b)\right)=2$ and the points $b$ and $g^{3 k}(b)$ are separated by $g^{2 k}(b)$ and $g^{k}(b)$, or $\operatorname{Val}_{C}\left(g^{2 k}(b)\right)=1$ and they are separated by $g^{k}(b)$. In both cases, $b$ and $g^{3 k}(b)$ are separated by $g^{k}(b)$. Iterating the previous argument we get that, for every $2 \leq m<r$, the points $b$ and $g^{m k}(b)$ are separated by $g^{k}(b)$. In consequence, $g^{k}(b) \in\left(g^{(r-1) k}(b), b\right) \cap P=\left(g^{(r-1) k}(b), g^{r k}(b)\right) \cap P$, in contradiction with (21).

To prove the "if" part of the statement of Theorem E we need to introduce a slight generalization of the notion of a monotone interval map. Let $f: X \longrightarrow Y$ be a continuous map. We say that $f$ is monotone if $f^{-1}(K)$ is a connected subset of $X$ for every connected subset $K$ of $Y$. To understand why this definition extends the notion of monotonicity on an interval to trees see Figure 8.

It seems reasonable that a tree map $f: T \longrightarrow T$ that is monotone has entropy 0 . To prove this fact, we use the classical notion of horseshoe and Theorem B of [10], which links the positive entropy of a graph map (in particular, a tree map) to the existence of horseshoes. For $s \geq 2$, we say that a graph map $f: G \longrightarrow G$ has an s-horseshoe if there is an interval $I \subset G$ and $s$ subintervals of $I$ with pairwise disjoint interiors, each of them mapped by $f$ onto the whole $I$.

Theorem 5.3 (Theorem B of [10]). If a continuous map $f$ of a graph into itself has positive topological entropy then there exists sequences $\left(k_{n}\right)_{n=1}^{\infty}$ and $\left(s_{n}\right)_{n=1}^{\infty}$ of positive integers such that for each $n$ the map $f^{k_{n}}$ has an $s_{n}$-horseshoe and

$$
\limsup _{n \rightarrow \infty} \frac{1}{k_{n}} \log \left(s_{n}\right)=h(f) .
$$

The fact that a monotone map of a tree has zero entropy is now a direct consequence of Theorem 5.3:

Corollary 5.4. Let $T$ be a tree and let $f: T \longrightarrow T$ be a monotone map. Then, $h(f)=0$.

Proof. From the definition of monotonicity, it directly follows that $f^{k}$ is a monotone map for every $k \geq 1$. On the other hand, assume that a map has an $s$-horseshoe given by an interval $I$ and $s \geq 2$ subintervals $I_{1}, I_{2}, \ldots, I_{s}$ of $I$. Let $x \in I_{1}$. From 

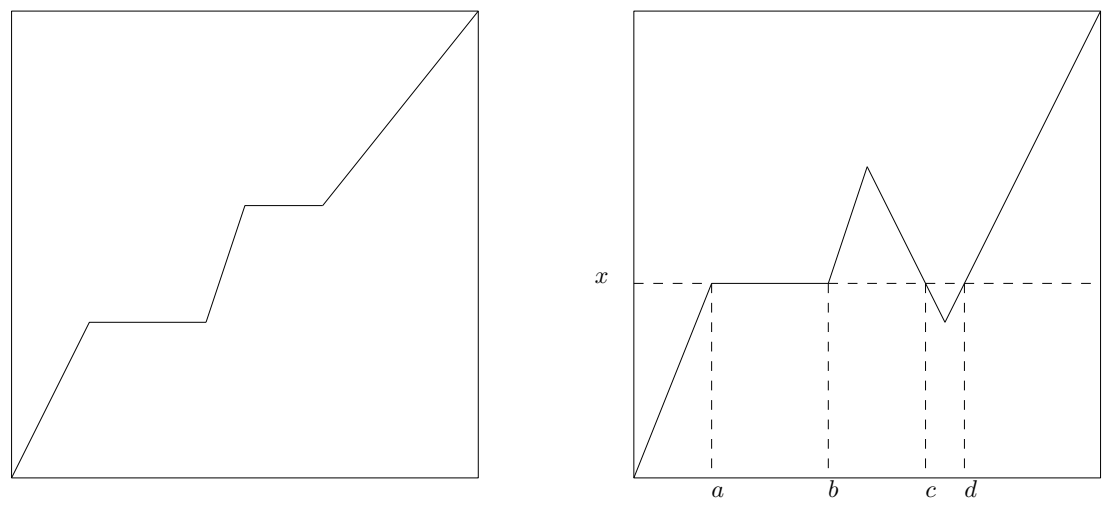

FIGURE 8. Left: a monotone interval map, for which the preimage of any connected set is also connected. Right: a non-monotone interval map, for which $f^{-1}(x)=[a, b] \cup\{c\} \cup\{d\}$, a disconnected set.

the definition of horseshoe it easily follows that $f^{-1}(x)$ has at least $s$ connected components and, thus, is not connected. Therefore, a map with horseshoes cannot be monotone. Collecting it all, we conclude that for every $k \geq 1$, the map $f^{k}$ has no horseshoes. Then, the corollary follows from Theorem 5.3.

Observe that if $(T, P, f)$ is a monotone model then, for every interval $[a, b] \subset T$ such that $(a, b) \cap P=\emptyset, f$ is monotone from $[a, b]$ to $[f(a), f(b)]$. In fact this is also true when one replaces $[a, b]$ by the convex hull of any subset of a discrete component of $(T, P)$ as the following lemma shows.

Lemma 5.5. Let $(T, P, f)$ be a monotone model and let $C$ be a subset of a discrete component of $(T, P)$. Then, $f$ is a monotone map from $\langle C\rangle$ to $\langle f(C)\rangle$.

Proof. Since $f$ is $P$-monotone, it follows that $f(\langle C\rangle)=\langle f(C)\rangle$. Let $K$ be a connected subset of $\langle f(C)\rangle$. It is well known that the connected subsets of a tree are trees. Hence, $K \backslash(P \cup V(T))$ is the union of a finite number of pairwise disjoint open intervals $I_{1} \cup I_{2} \cup \ldots \cup I_{k}$. Since $I_{i} \cap P=\emptyset$ and $f$ is $P$-monotone, $f^{-1}\left(\mathrm{Cl}\left(I_{i}\right)\right)$ is connected for every $1 \leq i \leq k$. It follows that, when $\mathrm{Cl}\left(I_{i}\right) \cap \mathrm{Cl}\left(I_{j}\right) \neq \emptyset$, $f^{-1}\left(\mathrm{Cl}\left(I_{i}\right) \cup \mathrm{Cl}\left(I_{j}\right)\right)$ is connected. Then, a simple iterative argument yields that $f^{-1}(K)$ is connected and the lemma follows.

The following proposition is the main tool for proving Theorem E.

Proposition 5.6. Let $\mathcal{P}$ be an n-periodic pattern. Then, $h(\mathcal{P})=0$ if and only if either $\mathcal{P}$ is trivial or has a separated p-block structure with trivial blocks such that the associated skeleton has entropy 0.

Proof. Let $(T, P, f)$ be the canonical model of $\mathcal{P}$. We start by proving the "if" part of the statement. If $\mathcal{P}$ is trivial, then we already know that $h(\mathcal{P})=0$. Assume that $\mathcal{P}$ is not trivial and that there exists a separated $p$-block structure $P=P_{1} \cup P_{2} \cup \ldots \cup P_{p}$ such that the associated skeleton has entropy 0 and the patterns $\left(\left[\left\langle P_{i}\right\rangle, P_{i}\right],\left[\left.f^{p}\right|_{P_{i}}\right]\right)$ are trivial for each $1 \leq i \leq p$. By Lemma 2.1, $f\left(\left\langle P_{i}\right\rangle\right)=\left\langle P_{i+1}\right\rangle$ for $1 \leq i<p$ and $f\left(\left\langle P_{p}\right\rangle\right)=\left\langle P_{1}\right\rangle$. On the other hand, since the pattern $\left(\left[\left\langle P_{i}\right\rangle, P_{i}\right],\left[\left.f^{p}\right|_{P_{i}}\right]\right)$ is trivial, $P_{i}$ is a subset of a discrete component of $(T, P)$. Therefore, Lemma 5.5 tells us that $\left.f\right|_{\left\langle P_{i}\right\rangle}$ for $1 \leq i<p$ and $\left.f\right|_{\left\langle P_{p}\right\rangle}$ are monotone maps. From the definition of monotonicity it follows that $\left.f^{p}\right|_{\left\langle P_{i}\right\rangle}$ is monotone for 
every $1 \leq i \leq p$. Then, from Corollary $5.4, h\left(\left.f^{p}\right|_{\left\langle P_{i}\right\rangle}\right)=0$ for $1 \leq i \leq p$. In consequence, $h(\mathcal{P})=0$ by Theorem D.

Next we prove the "only if" part of the statement. Assume that $\mathcal{P}$ is not trivial and $h(\mathcal{P})=0$. By Theorem 5.1, $\mathcal{P}$ is strongly reducible. In particular, there exists a basic path $\pi$ of $(T, P)$ such that $\mathcal{P}$ is $\pi$-reducible. Let $\mathcal{C}=\left\{C_{1}, C_{2}, \ldots, C_{p}\right\}$ be the set of connected components of $\bigcup_{i>0}\left\langle f^{i}(\pi)\right\rangle$. Then, $f$ acts as a $p$-cyclic permutation of $\mathcal{C}$. Let $P_{i}:=P \cap C_{i}$ for $1 \leq \bar{i} \leq p$. Since $C_{i}=\left\langle P_{i}\right\rangle$, Proposition 5.2 tells us that $\left(\left[\left\langle P_{i}\right\rangle, P_{i}\right],\left[\left.f^{p}\right|_{P_{i}}\right]\right)$ are trivial patterns for all $1 \leq i \leq p$. Observe that $p>1$ because otherwise $C_{1}=T, C_{1} \cap P=P$ and in this case $\mathcal{P}$ would be a trivial pattern; a contradiction. Hence, since all sets $\left\langle P_{i}\right\rangle$ are pairwise disjoint, the partition $P=P_{1} \cup P_{2} \cup \ldots \cup P_{p}$ defines a separated $p$-block structure for $\mathcal{P}$. Since $h(\mathcal{P})=0$, from Theorem $\mathrm{D}$ we get that the entropy of the associated skeleton is 0 .

Now we are ready to prove Theorem E.

Proof of Theorem E. The "only if" part of the statement follows directly by iteratively using Proposition 5.6 finitely many times.

Now assume that $\mathcal{P}$ is $k$-starry for some $k \geq 1$. We have to show that $h(\mathcal{P})=0$. We will proceed by induction on $k$. If $k=1$, then $\mathcal{P}$ is trivial by definition and thus $h(\mathcal{P})=0$. Assume now that any $k^{\prime}$-starry pattern has entropy zero for every $1 \leq k^{\prime}<k$. Let $(T, P, f)$ be the canonical model of $\mathcal{P}$. Since $\mathcal{P}$ is $k$-starry, there is a partition $P=P_{1} \cup P_{2} \cup \ldots \cup P_{p}$ defining a separated $p$-block structure with trivial blocks whose associated skeleton $\mathcal{Q}$ is $(k-1)$-starry. By the induction hypothesis, $h(\mathcal{Q})=0$. On the other hand, since each $P_{i}$ is a subset of a discrete component of $(T, P)$, we get that $h\left(\left.f^{p}\right|_{\left\langle P_{i}\right\rangle}\right)=0$ for $1 \leq i \leq p$, as in the proof of the "if" part of Proposition 5.6. In consequence, $h(\mathcal{P})=0$ by Theorem D.

Finally we prove statements (a) and (b) of Theorem F. It is clear that both results derive immediately from the following proposition and corollary.

Proposition 5.7. Let $\mathcal{P}$ be an n-periodic pattern and let $(T, P, f)$ be the canonical model of $\mathcal{P}$. Let $k \in \mathbb{N}$ be relatively prime to $n$. If $h(\mathcal{P})=0$ then $h\left(\left([T, P],\left[f^{k}\right]\right)\right)=$ 0 .

Proof. Set $\mathcal{Q}=\left([T, P],\left[f^{k}\right]\right)$. We have to prove that $h(\mathcal{Q})=0$. This clearly holds when $\mathcal{P}$ is trivial, since in this case $\mathcal{Q}$ is also trivial.

Now assume that $\mathcal{P}$ is not trivial. We claim that for some $p<n$ there exist two $p$-periodic patterns $\mathcal{P}^{\prime}=\left(\left[T^{\prime}, P^{\prime}\right],\left[f^{\prime}\right]\right)$ and $\mathcal{Q}^{\prime}=\left(\left[T^{\prime}, P^{\prime}\right],\left[\left(f^{\prime}\right)^{k}\right]\right)$ such that $h\left(\mathcal{P}^{\prime}\right)=0$ and $h\left(\mathcal{Q}^{\prime}\right)=h(\mathcal{Q})$. It is clear that the proposition follows from the iterative use of this claim together with the remark in the first paragraph of the proof.

Now let us prove the claim. By Theorem E, there exists a $p$-block structure $P=P_{1} \cup P_{2} \cup \ldots \cup P_{p}$ such that the associated skeleton has entropy 0 and the patterns $\left(\left[\left\langle P_{i}\right\rangle, P_{i}\right],\left[\left.f^{p}\right|_{P_{i}}\right]\right)$ are trivial (in consequence, have entropy 0 ) for each $1 \leq i \leq p$. Let $\left(\left[T^{\prime}, P^{\prime}\right],\left[f^{\prime}\right]\right)$ be the associated skeleton. Now observe that, since $k$ and $n$ are relatively prime, $f^{k}$ acts as a $p$-cyclic permutation on the set $\left\{P_{1}, P_{2}, \ldots, P_{p}\right\}$ (in general, different from the one induced by $f$ ). It follows that $P_{1} \cup P_{2} \cup \ldots \cup$ $P_{p}$ also defines a $p$-block structure for $\mathcal{Q}$, and that the associated skeleton $\mathcal{Q}^{\prime}$ is $\left(\left[T^{\prime}, P^{\prime}\right],\left[\left(f^{\prime}\right)^{k}\right]\right)$. By Theorem D,

$$
h(\mathcal{Q})=\max \left\{h\left(\mathcal{Q}^{\prime}\right),(1 / p) h\left(\left.f^{k p}\right|_{\left\langle P_{i}\right\rangle}\right)\right\} \text { for any } 1 \leq i \leq p .
$$

Since the pointed trees $\left(\left\langle P_{i}\right\rangle, P_{i}\right)$ have one discrete component, using Lemma 5.5 as in the proof of Proposition 5.7 yields that $\left.f^{k p}\right|_{P_{i}}$ is a monotone map. Then, by 
Corollary 5.4, $h\left(\left.f^{k p}\right|_{P_{i}}\right)=0$. Hence, from (22) it follows that $h\left(\mathcal{Q}^{\prime}\right)=h(\mathcal{Q})$. This ends the proof of the claim and proves the proposition.

Corollary 5.8. Let $\mathcal{P}$ be an $n$-periodic pattern and let $(T, P, f)$ be the canonical model of $\mathcal{P}$. Let $k \in \mathbb{N}$ be relatively prime to $n$. If $h\left(\left([T, P],\left[f^{k}\right]\right)\right)=0$ then $h(\mathcal{P})=0$.

Proof. Since $n$ and $k$ are relatively prime, there exists $k^{\prime}$ such that $k k^{\prime}$ is congruent to 1 modulo $n$. Therefore, $\left([T, P],\left[\left(f^{k}\right)^{k^{\prime}}\right]\right)=([T, P],[f])=\mathcal{P}$. Then, since $\left([T, P],\left[f^{k}\right]\right)$ is an $n$-periodic pattern with zero entropy and $n$ and $k^{\prime}$ are also relatively prime, $h(\mathcal{P})=0$ by Proposition 5.7.

\section{REFERENCES}

[1] R. L. Adler, A. G. Konheim, and M. H. McAndrew, Topological entropy, Trans. Amer. Math. Soc. 114 (1965), 309-319. MR 0175106 (30 \#5291)

[2] Ll. Alsedà and X. Ye, Division for star maps with the branching point fixed, Acta Math. Univ. Comenian. (N.S.) 62 (1993), no. 2, 237-248. MR 1270511 (95e:58057)

[3] Ll. Alsedà, J. Los, F. Mañosas, and P. Mumbrú, Canonical representatives for patterns of tree maps, Topology 36 (1997), no. 5, 1123-1153. MR 1445556 (99f:58062)

[4] Ll. Alsedà, J. Llibre, and M. Misiurewicz, Combinatorial dynamics and entropy in dimension one, second ed., Advanced Series in Nonlinear Dynamics, vol. 5, World Scientific Publishing Co. Inc., River Edge, NJ, 2000. MR 1807264 (2001j:37073)

[5] Ll. Alsedà and X. Ye, No division and the set of periods for tree maps, Ergodic Theory Dynam. Systems 15 (1995), no. 2, 221-237. MR 1332401 (96d:58109)

[6] L. Block, J. Guckenheimer, M. Misiurewicz, L.S. Young, Periodic points and topological entropy of one-dimensional maps, Global theory of dynamical systems, pp. 18-34, SLNM 819, Springer, Berlin.

[7] L. Block, Simple periodic orbits of mappings of the interval, Trans. Amer. Math. Soc. 254 (1979), 391-398. MR 539925 (80m:58031)

[8] F.R. Gantmacher, The theory of matrices, AMS Chelsea Publishing Company, New York 1989-90 (2nd edition).

[9] T.Y. Li, M. Misiurewicz, G. Pianigiani, and J.A. Yorke, No division implies chaos, Trans. Amer. Math. Soc. 273 (1982), no. 1, 191-199. MR 664037 (83i:28024)

[10] J. Llibre, M. Misiurewicz, Horseshoes, entropy and periods for graph maps, Topology 32 (1993), no. 3, 649-664.

[11] W. Rudin, Principles of mathematical analysis, McGraw-Hill, Singapore, 1976.

[12] O. M. Sharkovskii, Co-existence of cycles of a continuous mapping of the line into itself, Ukrain. Mat. Z̆. 16 (1964), 61-71. MR 0159905 (28 \#3121)

[13] W. P. Thurston, On the geometry and dynamics of diffeomorphisms of surfaces, Bull. Amer. Math. Soc. (N.S.) 19 (1988), 417-431. MR 956596 (89k:57023)

[14] R.S. Varga, Matrix iterative analysis, expanded ed., Springer Series in Computational Mathematics, vol. 27, Springer-Verlag, Berlin,2000. MR 1753713 (2001g:65002)

Departament de Matemàtiques, Edifici Cc, Universitat Autònoma de Barcelona, 08913 Cerdanyola del Vallès, Barcelona, Spain

E-mail address: alseda@mat.uab.cat

Departament d'Informàtica i Matemàtica Aplicada, Universitat de Girona, Lluís SanTALÓ S/N, 17071 GiRONA, SPAIN

E-mail address: juher@ima.udg.edu

Departament de Matemàtiques, Edifici Cc, Universitat Autònoma de Barcelona, 08913 Cerdanyola del Vallès, Barcelona, Spain

E-mail address: manyosas@mat.uab.cat 